\title{
On Regular Fréchet-Lie Group of Invertible Inhomogeneous Fourier Integral Operators on $\mathbf{R}^{n}$
}

\author{
Naoya MIYAZAKI \\ Science University of Tokyo \\ (Communicated by Y. Maeda)
}

\section{Introduction.}

The fundamental solution of the Schrödinger equation on $\mathbf{R}^{n}$

$$
\frac{d}{d t} \Phi_{t}=\frac{\sqrt{-1}}{\hbar} \hat{H}_{t} \Phi_{t}, \quad \Phi_{0}=\mathrm{id}
$$

for a time-dependent Hamiltonian operator $\hat{H}_{t}$ is heuristically described as the so-called Feynman path integral (cf. [FH]):

$$
\int_{\Omega(t, x, y)} \exp \frac{i}{\hbar} S(t, \gamma) \mathscr{D}[\gamma],
$$

where $\Omega(t, x, y)$ is the space of all paths from $x$ to $y$ in time $t . \mathscr{D}[\gamma]$ is a "measure" on $\Omega(t, x, y)$, although it is not a genuine measure. Several mathematical interpretations have been examined by many authors (cf. [F2], [F3], [Ki], [KK], [AH], [IM], [N1]), and (1.2) is understood as an idealized expression of a product integral in a certain class of linear operators. Another treatment is seen in [I1], [I2].

The convergence of product integrals are shown in these literature under the uniform topology for operators, the pointwise convergence topology of amplitude and phase functions ([F2], [Ki]) or Fréchet topology of function spaces of amplitude and phase functions ([F3], [KK]).

However, it would be natural to define the notion of product integrals on a certain "Lie group": We should define an infinite dimensional Lie group $G$ so that (1.1) can be understood as the equation of an integral curve for a time-dependent right-invariant vector field $X_{t}$, defined by $X_{t}=(\sqrt{-1} / \hbar) \hat{H}_{t}$. Furthermore the solution is given by the product integral on $G$.

Indeed, this is accomplished in the Banach-Lie group. The solution of the equation

Received May 28, 1994 
$(d / d t) \Phi_{t}=X_{t}\left(\Phi_{t}\right)$ is described as the product integral $\Phi_{t}=\prod_{0}^{t} \exp X_{\tau} d \tau$ on $G$ (cf. [N2]).

In this paper, we shall construct a certain Fréchet-Lie group $G$ on which the equation (1.1) can be regarded as the equation of integral curve of a vector field on $G$, and whose solution can be obtained by the product integral.

A Fréchet-Lie group is by definition a $C^{\infty}$ Fréchet manifold with a compatible smooth group structure. Note, however, that fundamental theorems such as implicit function theorem and the existence of solutions of ordinary differential equations do not hold for Fréchet manifolds in general.

A Fréchet-Lie group $G$ is said to be regular if the product integrals are well-defined on $G$ (see Definition 2.2).

It is known (cf. [F2], [F3], [Ki], [KK]) that the fundamental solution of (1.1) is given by a certain class of invertible inhomogeneous Fourier integral operators of order 0 written in the form:

$$
(2 \pi \hbar)^{-n} \iint a(\bar{x}, \xi) e^{(\sqrt{-1} / \hbar)\{S(\bar{x}, \xi)-\xi \cdot x\}} u(x) d x d \xi,
$$

where $\xi \cdot x=\sum_{i=1}^{n} \xi_{i} \cdot x_{i}$.

We choose such phase function $S$ as $S=S_{\tilde{\varphi}}+f$, where $S_{\tilde{\phi}}$ and $f$ is a homogeneous function on $\mathbf{R}^{2 n}-\{0\}$ of degree 2 and degree 1 respectively. $S_{\tilde{\varphi}}$ generates a contact transformation $\tilde{\varphi}$ on the unit sphere $S^{2 n-1}$ in $\mathbf{R}^{2 n}$ in a similar manner to symplectic geometry. Here, we assume that $\tilde{\varphi}$ is sufficiently close to the identity. The amplitude function $a(x, \xi)$ is a complex valued function on $\mathbf{R}^{2 n}$ with the asymptotic expansion

$$
a(x, \xi) \sim a_{0}(\omega)+a_{-1}(\omega) \rho^{-1}+a_{-2}(\omega) \rho^{-2} \cdots,
$$

for a large $\rho$, where

$$
\rho=\sqrt{x_{1}^{2}+\cdots+x_{n}^{2}+\xi_{1}^{2}+\cdots+\xi_{n}^{2}}, \quad \omega \in S^{2 n-1}, \quad(x, \xi)=\rho \omega .
$$

We shall denote (1.3) by

$$
(F(a, f, \tilde{\varphi}) u)(\bar{x})=\iint a(\bar{x}, \xi) e^{(\sqrt{-1} / \hbar)(S \tilde{\boldsymbol{\varphi}}(\bar{x}, \xi)+f(\bar{x}, \xi)-\xi \cdot x\}} u(x) d x d \xi,
$$

where $d x=(2 \pi \hbar)^{-n / 2} d x$ etc.

If $S_{\tilde{\varphi}}=\bar{x} \cdot \xi$ and $f=0$, then $\tilde{\varphi}$ is the identity and then $F(a, 0$, id) in (1.6) is an ordinary pseudo-differential operator with the symbol $a(\bar{x}, \xi)$ in the symbol class $S_{1,0}^{0}$, but if $f \neq 0$ then $F\left(a, f\right.$, id) is a pseudo-differential operator with the symbol $a(\bar{x}, \xi) e^{(\sqrt{-1} / h) f(\bar{x}, \xi)}$ which is in the symbol class $S_{0,0}^{0}$ (cf. [Ku] for the notations $S_{0,0}^{0}, S_{1,0}^{0}$ etc.).

Using the above notations, we set $G F^{0}$ as the group generated by inhomogeneous Fourier integral operators $F(a, f, \tilde{\varphi})$ given in (1.6) such that $a$ (resp. $f$ ) are close to 1 (resp. 0) and $\tilde{\varphi}$ is close to the identity in the $C^{\infty}$ topology. (To be precise, see §3.)

The main theorem of this paper is stated as follows: 
THEOREM A. $G F^{0}$ is a regular Fréchet-Lie group with the Lie algebra $\mathscr{G}$ consisting of $C^{\infty}$ functions on $\mathbf{R}^{2 n}$ with asymptotic expansions:

$$
\sqrt{-1} a_{2}(\omega) \rho^{2}+\sqrt{-1} a_{1}(\omega) \rho+a_{0}(\omega)+a_{-1}(\omega) \rho^{-1}+a_{-2}(\omega) \rho^{-2}+\cdots,
$$

where $a_{2}, a_{1}$ are real valued smooth functions and $a_{0}, a_{-1}, a_{-2}, \cdots$ are complex valued smooth functions.

The above theorem involves the convergence of product integrals. Hence, the fundamental solution of the Schrödinger equation (1.1) such that $(\sqrt{-1} / \hbar) \hat{H}_{t} \in \mathscr{G}$ is given as an element of $G F^{0}$ by the product integral $\Phi_{t}=\prod_{0}^{t} \exp X_{\tau} d \tau$.

Note that the topology of $G F^{0}$ is given by using the $C^{\infty}$ topology on each homogeneous functions. Hence the topology of $G F^{0}$ is much stronger than the operator topology and the pointwise convergence topology of amplitude and phase functions.

As the statement of Theorem A is similar to that of [OMY]-I II, the difference between $G F^{0}$ and the group $G \mathscr{F}^{0}$ which is constructed in [OMY]-I $\sim$ II and $[\mathrm{OMYK}]-\mathrm{I} \sim \mathrm{IV}$ is mentioned below, although the main idea of the proof is given by their papers:

\begin{tabular}{|c|c|c|}
\hline & $G^{\circ}{ }^{0}[\mathrm{OMYK}]$ & $G F^{\mathbf{0}}$ \\
\hline base manifold & $\begin{array}{l}\text { compact Riemann } \\
\text { manifold } N\end{array}$ & non-compact $\mathbf{R}^{n}$ \\
\hline compactification & cosphere bundle $S^{*} N$ & $S^{2 n-1}$ \\
\hline phase function & $\begin{array}{l}\text { homogenous functions } \\
\phi(x, \xi) \text { of degree } 1 \\
\text { with respect to } \xi\end{array}$ & $\begin{array}{l}\text { summation of homogeneous } \\
\text { functions } \phi_{2}(x, \xi) \text { and } \phi_{1}(x, \xi) \\
\text { of degree } 2 \text { and } 1 \text { with respect } \\
\text { to }(x, \xi) \text { with certain conditions } \\
\text { (cf. (A-II) of [AF]) }\end{array}$ \\
\hline equation & relative Schrödinger eq. & non-relative Schrödinger eq. \\
\hline
\end{tabular}

ACKnowledgements. The author would like to thank Professors Y. Maeda, A. Yoshioka and H. Omori for their helpful comments and advices.

\section{Several remarks on regular Fréchet-Lie groups.}

Throughout this paper, we will use the notion of differentiability on a Fréchet space as usual (cf. [L] for instance), but we recall the partial derivations as follows:

Let $\mathbf{E}, \mathbf{F}$ and $\mathbf{G}$ be Fréchet spaces, $U, V$ be open subsets of $\mathbf{E}, \mathbf{F}$ respectively and let $f: U \times V \rightarrow \mathbf{G}$ be a mapping. 
DEFINITION 2.1. $f$ is called a partial $C^{r}$-mapping with respect to the first variable iff for each fixed $v \in V, f(\cdot, v): U \rightarrow G$ is a $C^{r}$-mapping and $i$-th derivatives with respect to the first variable

$$
D_{1}^{i} f: U \times V \times \overbrace{\mathbf{E} \times \cdots \times \mathbf{E}}^{i} \rightarrow \mathbf{G} \quad(i=1,2, \cdots, r)
$$

are continuous mappings.

Note that the continuity with respect to " $(u, v)$ " is requested.

The partial derivatives for the second variable is defined similarly and are denoted by $D_{2}^{i} f$.

The following properties of $C^{r}$-mappings are well-known. (Cf. [L] for the proof.)

Proposition 2.2. (I) Any continuous p-linear mapping $f: \mathbf{E} \times \cdots \times \mathbf{E} \rightarrow \mathbf{F}$ is $C^{\infty}$ such that $D^{p+1} f=0$.

(II) Composition $f \circ g$ of $C^{r}$-mappings $f$ and $g$ is also a $C^{r}$-mapping.

(III) If $f: U \rightarrow \mathbf{F}$ is a $C^{r}$-mapping, then

$$
\left(D^{i} f\right): U \times \overbrace{\mathbf{E} \times \cdots \times \mathbf{E}}^{i} \rightarrow \mathbf{F}
$$

is a $C^{r-i}$-mapping for $i=0,1,2, \cdots, r$, and

$$
D_{1}^{j} D^{i} f=D^{i+j} f, \quad \text { for } i+j \leq r .
$$

Conversely, if $f: U \rightarrow \mathbf{F}$ is a $C^{i}$-mapping for some $i \leq r$ and

$$
\left(D^{i} f\right): U \times \overbrace{\mathbf{E} \times \cdots \times \mathbf{E} \rightarrow \mathbf{F}}^{i}
$$

is $C^{r-i}$, then $f$ is $C^{r}$.

(IV) $f: U \times V \rightarrow \mathbf{G}$ is $C^{r}$ if and only if $f$ is $C^{r}$ with respect to both first and second variables.

By using the notion of differentiability, $C^{\infty}$ Fréchet manifolds, and Fréchet-Lie group are defined in the same manner as in finite dimensional cases.

We now recall the notion of regular Fréchet-Lie groups ([OMYK]-IV):

Let $G$ be a Fréchet-Lie group and let $h(s ; t):[0, \varepsilon] \times[a, b] \rightarrow G$ be a $C^{1}$ mapping such that $h(0 ; t) \equiv$ identity of $G$ for some $\varepsilon>0$ and a compact interval $[a, b]$. Let $\Delta^{(n)}: t_{k_{n}-1}^{(n)}<s \leq t_{k_{n}}^{(n)}<\cdots<t_{l_{n}}^{(n)}<t \leq t_{l_{n}+1}^{(n)}$ be divisions of interval $[s, t] \subset[a, b]$ such that $\left|\Delta^{(n)}\right|=\max _{i}\left|t_{i}^{(n)}-t_{i-1}^{(n)}\right| \rightarrow 0(n \rightarrow \infty)$, and set the product

$$
\prod_{s}^{t}\left(h ; \Delta^{(n)}\right)=h\left(t-t_{l_{n}}^{(n)} ; t_{l_{n}}^{(n)}\right) \cdot h\left(t_{l_{n}}^{(n)}-t_{l_{n}-1}^{(n)} ; t_{l_{n}-1}^{(n)}\right) \cdots h\left(t_{k_{n}}^{(n)}-s ; s\right) .
$$

DEFINITION 2.3. A Fréchet-Lie group $G$ will be called a regular Fréchet-Lie group 
iff for any $h$, the product (2.1) converges uniformly for $s, t \in[a, b]$ with respect to the uniform topology on $G$ as $\left|\Delta^{(n)}\right| \rightarrow 0(n \rightarrow \infty)$.

The limit of $\left\{\prod_{s}^{t}\left(h ; \Delta^{(n)}\right)\right\}_{n=1}^{\infty}$ is written by $\prod_{s}^{t}(h ; d \tau)$ and it is called the product integral.

Remark that any Fréchet space is an abelian regular Fréchet-Lie group, because the product integral is the ordinary integral on an interval. Several fundamental properties and examples of regular Fréchet-Lie groups are shown in [OMY]-I II, [OMYK]-III $\sim$ VIII. For example, $\mathscr{D}_{\omega}\left(S^{2 n-1}\right)$, the group of all contact transformations with respect to the standard contact 1 -form $\theta$ on $S^{2 n-1}$, is known to be a regular Fréchet-Lie group in the paper [Om], [OMYK]-II.

As is seen in the above definition, the notion of product integrals can be defined on local Fréchet-Lie groups. It is easy to see the following:

LEMMA 2.4. Topological group $G$ is a regular Fréchet-Lie group if and only if its universal covering group $\widetilde{G}$ is a regular Fréchet-Lie group.

Obviously, the direct product $G \times H$ of regular Fréchet-Lie groups $G, H$ is a regular Fréchet-Lie group. A little calculation shows also that a semi-direct product $G \succ_{\varphi} H$ with a $C^{\infty}$ action $\varphi$ of $G$ onto $H$ as an automorphism group is a regular Fréchet-Lie group. More generally, the following lemma is known (cf. [OMYK]-III):

LEMMA 2.5. Given a short exact sequence of Fréchet-Lie groups:

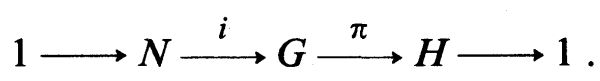

\section{Suppose}

(1) $N$ is a closed normal regular Fréchet-Lie group (with respect to the relative topology),

(2) $H$ is a regular Fréchet-Lie group,

(3) $i, \pi$ are smooth,

(4) there exists a local section $j$, that is, $j$ is a $C^{\infty}$-mapping of an open neighborhood $U_{\tilde{e}}$ of the identity $\tilde{e}$ of $H$ into $G$ such that $\pi \circ j=\operatorname{Id}_{U \tilde{e}}$.

Then $G$ is also a regular Fréchet-Lie group.

We shall use the above lemma successively for the proof of Theorem A. Note that the above lemma can be applied, even if the projection $\pi$ in (2.2) is not a surjective homomorphism, but a local homomorphism which covers a neighborhood of the identity of $H$. This is because that if $\pi: U_{e} \rightarrow H$ is a local homomorphism defined on a neighborhood $U_{e}$ of the identity $e$ of $G$ and the image contains a neighborhood of the identity $\tilde{e}$ of $H$, then there exists a homomorphism $\tilde{\pi}$ of the universal covering group $\tilde{G}$ onto the identity component $\tilde{H}_{0}$ of the universal covering $\tilde{H}$ of $H$ such that $\pi$ may be identified with the restriction of $\tilde{\pi}$ on a neighborhood. 


\section{Outline of the proof.}

At first, we shall fix notations and spaces which will be used throughout this paper: With the quantity $\rho$ in (1.5), we set

$$
\langle x ; \xi\rangle=\sqrt{1+\rho^{2}} .
$$

Fix a cut off function $\kappa \in C_{\mathbf{R}}^{\infty}\left(\mathbf{R}^{2 n}\right)$ such that $0 \leq \kappa(x, \xi) \leq 1$ and

$$
\kappa(x, \xi)= \begin{cases}0 & \text { (if } \rho \leq 1 / 2) \\ 1 & \text { (if } \rho \geq 1) .\end{cases}
$$

Let $C_{\mathbf{K}}^{\infty}\left(S^{2 n-1}\right)$ be the space of all $\mathbf{K}(\mathbf{R}$ or $\mathbf{C})$-valued $C^{\infty}$ functions on the unit sphere $S^{2 n-1}$ of $\mathbf{R}^{2 n}$, and let $U_{i, \pm}$ 's be open sets on $S^{2 n-1}$ given by

$$
U_{i, \pm}=\left\{\left(\omega_{1}, \cdots, \pm\left\{1-\sum_{j \neq i} \omega_{j}^{2}\right\}^{1 / 2}, \cdots, \omega_{2 n}\right) ; 1-\sum_{j \neq i} \omega_{j}^{2}>\frac{1}{n+1}\right\}
$$

where $\left(\omega_{1}, \cdots, \omega_{i-1}, \omega_{i+1}, \cdots, \omega_{2 n}\right)$ are coordinate functions on $U_{i, \pm}$.

The space $H_{\mathbf{K}}^{-i}$ of all smooth homogeneous functions of degree $-i(i \in \mathbf{Z})$ is naturally identified with $C_{\mathbf{K}}^{\infty}\left(S^{2 n-1}\right) \rho^{-i} . H_{\mathbf{K}}^{-i}$ is a Fréchet space by the system of norms

$$
\|f\|_{k}=\sum_{|\gamma| \leq k} \max _{i, \pm} \sup _{U_{l, \pm}}\left|\partial^{\gamma} f\right|
$$

on $C_{\mathbf{K}}^{\infty}\left(S^{2 n-1}\right)$, where $\partial^{\gamma} f$ means the ordinary derivative of $f$ on $U_{i, \pm}$.

Definition 3.1. For any non-negative integer $L$, we define the space $\mathfrak{B}_{\mathbf{K}}^{-L}$ as follows:

$$
\mathfrak{B}_{\mathbf{K}}^{-L}=\left\{r \in C_{\mathbf{K}}^{\infty}\left(\mathbf{R}^{2 n}\right)\left|\forall \alpha, \beta, \exists C_{\alpha, \beta} ;\langle x ; \xi\rangle^{L}\right| \partial_{x}^{\alpha} \partial_{\xi}^{\beta} r(x, \xi) \mid \leq C_{\alpha, \beta}\right\} .
$$

This is a Fréchet space with a system of norms:

$$
\|r\|_{-L, k}=\sum_{|\alpha|+|\beta| \leq k} \sup _{(x, \xi)}\langle x ; \xi\rangle^{L}\left|\partial_{x}^{\alpha} \partial_{\xi}^{\beta} r(x, \xi)\right| .
$$

Consider the space

$$
\Sigma_{\mathbf{K}}^{-m,-M}=H_{\mathbf{K}}^{-m} \oplus H_{\mathbf{K}}^{-m-1} \oplus \cdots \oplus H_{\mathbf{K}}^{-M} \oplus \mathfrak{B}_{\mathbf{K}}^{-M-1} \quad(-M \leq-m) .
$$

For any $f=\sum_{i=m}^{M} f_{-i} \rho^{-i} \oplus r_{-M-1}(f) \in \Sigma_{\mathbf{K}}^{-m,-M}$, we define the norm $\llbracket f \rrbracket_{-M, k}(k \geq M)$ by

$$
\left\|f \rrbracket_{-M, k}=\sum_{i=m}^{M}\right\| f_{-i}\left\|_{k-i}+\right\| r_{-M-1}(f) \|_{-M-1, k-M-1} .
$$

$\Sigma_{\mathbf{K}}^{-m,-M}$ is a Fréchet space by the system of norms $\left\{\llbracket f \rrbracket_{-M, k}\right\}_{k}$. Note that the family $\left\{\Sigma_{\mathbf{K}}^{-m,-M}, i_{-M}^{-M-1}\right\}$ forms a projective system of Fréchet spaces, where $i_{-M}^{-M-1}$ is the inclusion mapping $i_{-M}^{-M-1}: \Sigma_{\mathbf{K}}^{-m,-M-1} \rightarrow \Sigma_{\mathbf{K}}^{-m,-M}$ given by 


$$
i_{-M}^{-M-1}\left(\sum_{i=m}^{M+1} f_{-i} \rho^{-i} \oplus r_{-M-2}\right)=\sum_{i=m}^{M} f_{-i} \rho^{-i} \oplus\left\{\kappa f_{-M-1} \rho^{-M-1}+r_{-M-2}\right\}
$$

By $\Sigma_{\mathbf{K}}^{-m}$ we denote the projective limit $\lim _{M} \Sigma_{\mathbf{K}}^{-m,-M}$ with the projective limit topology.

Associating with this system, we have the following:

LEMMA 3.2. The projective limit space $\Sigma_{\mathbf{K}}^{-m}$ is identified with

$$
\begin{aligned}
\mathfrak{U}_{\mathbf{K}}^{-m}=\left\{f \in C_{\mathbf{K}}^{\infty}\left(\mathbf{R}^{2 n}\right) \mid\right. & \forall M \geq m, \exists f_{-m}, \cdots, f_{-M} \in C_{\mathbf{K}}^{\infty}\left(S^{2 n-1}\right) \\
& \text { such that } \left.f-\kappa\left(\sum_{i=m}^{M} f_{-i} \rho^{-i}\right) \in \mathfrak{B}_{\mathbf{K}}^{-M-1}\right\} .
\end{aligned}
$$

Proof. Any element $\left\{\sum_{i=m}^{M} f_{-i} \rho^{-i} \oplus r_{-M-1}\right\}_{M>m-1}$ of $\Sigma_{\mathbf{K}}^{-m}$ is written as

$$
r_{-m} \leftarrow f_{-m} \rho^{-m} \oplus r_{-m-1} \leftarrow \cdots \leftarrow \sum_{i=m}^{M} f_{-i} \rho^{-i} \oplus r_{-M-1} \leftarrow \cdots,
$$

where $r_{-m}=\kappa f_{-m} \rho^{-m}+r_{-m-1} \in \mathfrak{A}_{\mathbf{K}}^{-m}$.

Conversely, any element $f$ of $\mathfrak{U}_{\mathbf{K}}^{-m}$ corresponds to the element

$$
\left\{\sum_{i=m}^{M} f_{-i} \rho^{-i} \oplus\left(f-\kappa\left(\sum_{i=m}^{M} f_{-i} \rho^{-i}\right)\right)\right\}_{M}
$$

of $\Sigma_{\mathbf{K}}^{-m}$.

The following lemma is useful (cf. $[\mathrm{Ku}]$ ):

LEMMA 3.3. Let $g_{-i}(i=m, m+1, \cdots)$ be functions of $C^{\infty}\left(S^{2 n-1}\right)$. For any $M=m, m+1, \cdots$, and any $\delta>0$, there exists $g \in \Sigma_{\mathbf{K}}^{-m}$ such that

$$
\begin{gathered}
g-\kappa \sum_{i=m}^{M} g_{-i} \rho^{-i} \in \mathfrak{B}_{\mathbf{K}}^{-M-1}, \\
\llbracket g-\kappa \sum_{m}^{M} g_{-i} \rho^{-i} \rrbracket_{-M, M+1}<\delta .
\end{gathered}
$$

Proof. We shall choose $\varepsilon_{-j}$ such that

$$
g(x, \xi)=\sum_{j=m}^{m+N+1} \kappa g_{-j} \cdot r^{-j}+\sum_{j=m+N+2}^{\infty} \kappa_{\varepsilon_{-j}} g_{-j} \cdot r^{-j}
$$

satisfies (3.11), where $\kappa_{\varepsilon_{-j}}(x, \xi)=\kappa\left(\varepsilon_{-j}(x, \xi)\right)$.

First we take into account that

$$
\begin{gathered}
\kappa_{\varepsilon}(x, \xi) \rightarrow 0 \quad(\varepsilon \rightarrow 0, \text { uniform convergence on each compact set }), \\
\partial_{x}^{\alpha} \partial_{\xi}^{\beta} \kappa_{\varepsilon}(x, \xi) \rightarrow 0 \quad(\varepsilon \rightarrow 0, \text { uniformly, }|\alpha|+|\beta|>0), \\
\left|\partial_{x}^{\alpha} \partial_{\xi}^{\beta} \kappa_{\varepsilon}(x, \xi)\right| \leq C_{\alpha, \beta}\langle x ; \xi\rangle-|\alpha|-|\beta|
\end{gathered}
$$


Next we choose $\varepsilon_{-j}$ so that they satisfy

$$
\begin{gathered}
\varepsilon_{-j}>0, \quad \varepsilon_{-j}>\varepsilon_{-j-1}>\cdots \rightarrow 0, \\
C_{g_{-j}, \alpha, \beta} \cdot \varepsilon_{-j} \leq \delta \cdot 2^{-j-1} \quad(\forall \alpha, \beta ;|\alpha+\beta| \leq j) .
\end{gathered}
$$

Then we see

$$
\begin{gathered}
\text { if }\langle x ; \xi\rangle \leq \varepsilon_{-j}^{-1} \text { then } \kappa_{\varepsilon-j}(x, \xi)=0, \\
\text { if }\langle x ; \xi\rangle>\varepsilon_{-j}^{-1} \text { then } C_{g_{-j}, \alpha, \beta}\langle x ; \xi\rangle \leq C_{g_{-j}, \alpha, \beta} \varepsilon_{-j}
\end{gathered}
$$

Therefore

$$
\begin{aligned}
\left|\partial_{x}^{\alpha} \partial_{\xi}^{\beta}\left\{\kappa_{\varepsilon_{-j}}(x, \xi) \cdot g_{-j} \cdot r^{-j}\right\}\right| \leq C_{g_{-j}, \alpha, \beta}\langle x ; \xi\rangle^{-1}\langle x ; \xi\rangle^{-j-|\alpha+\beta|+1} \\
\quad \leq C_{g-j, \alpha, \beta} \varepsilon_{-j}\langle x ; \xi\rangle^{-j-|\alpha+\beta|+1} \leq \delta \cdot 2^{-j-1}\langle x ; \xi\rangle^{-j-|\alpha+\beta|+1}
\end{aligned}
$$

Thus we obtain

$$
\begin{aligned}
& \left|g(x, \xi)-\sum_{j=m}^{m+N+1} \kappa g_{-j} \cdot r^{-j}\right|_{m+N+1}^{(-m-N)} \\
& \leq\left|\sum_{j=m+N+2}^{\infty} \kappa_{\varepsilon-j} g_{-j} \cdot r^{-j}\right|_{m+N+1}^{(-m-N)} \leq \sup \langle x ; \xi\rangle^{m+N+1}\left\{\sum_{j=m+N+2}^{\infty}\left|\kappa_{\varepsilon-j} g_{-j} \cdot r^{-j}\right|\right\} \\
& \quad \leq \sup \langle x ; \xi\rangle^{m+N+1}\left\{\sum_{j=m+N+1}^{\infty} \delta 2^{-j-1}\langle x ; \xi\rangle^{-j}\right\} \leq \frac{\delta}{2} .
\end{aligned}
$$

For any $a \in \Sigma_{\mathbf{C}}^{0}$, we define the pseudo-differential operator $P(a)$ as follows:

$$
(P(a) u)(\bar{x})=O s-\iint a(\bar{x}, \xi) e^{(\sqrt{-1} / \hbar)((\bar{x}-x) \cdot \xi)} u(x) d x d \bar{d} \xi,
$$

where $O s-\iint$ means the oscillatory integral. As $P(a)$ corresponds uniquely to its symbol $a$, we identify $P(a)$ with $a$, and define the product $c=a * b$, iff $P(c)=P(a) \circ P(b)$, i.e.

$$
c(x, \xi)=O s-\iint a(x, \bar{\xi}) b(\bar{x}, \xi) e^{(\sqrt{-1} / \hbar)(x-\bar{x}) \cdot(\bar{\xi}-\xi)} d \bar{x} d \bar{\xi} .
$$

The following is well known (cf. $[\mathrm{Ku}])$ :

LEMMA 3.4. Under the notations as above, we have

$$
\begin{aligned}
& c(x, \xi)=\sum_{0 \leq|\alpha| \leq N} \frac{1}{\alpha !} \not D_{\xi}^{\alpha} a(x, \xi) \partial_{x}^{\alpha} b(x, \xi) \\
& \quad+\sum_{|\alpha|=N+1} \frac{N+1}{\alpha !} O s-\iiint(1-\theta)^{N} \not D_{\xi}^{\alpha} a(x, \xi+\theta \bar{\eta}) \partial_{x} b(x+\bar{y}, \xi) d \theta e^{-(\sqrt{-1} / \hbar) \bar{\eta} \cdot \bar{y}} \bar{d} \bar{y} \bar{d} \bar{\eta},
\end{aligned}
$$

where $\not_{\bar{\xi}}=(\hbar / \sqrt{-1}) \partial_{\bar{\xi}}$. 
The proof of Theorem A is based on the successive use of Lemma 2.5. So we first have to define several subgroups of $G F^{0}$.

If $a=1+h$ and $h$ is sufficiently close to 0 in $\Sigma_{\mathbf{C}}^{-m}(m=0,1,2, \cdots)$, then $P(1+h)$ in (3.18) is known to be invertible (cf. $\S 4)$. Let $G \Sigma^{-m}(m=0,1,2, \cdots)$ be the group of all pseudo-differential operators generated by $1+P(h)$ such that $h \in \Sigma_{\mathbf{C}}^{-m}$ and $h$ are sufficiently close to 0 so that $1+P(h)$ is invertible. Using Lemma 2.5, we have the following: (See $\S 4$ for the proof.)

Proposition A. $G \Sigma^{0}$ is a regular Fréchet-Lie group with the Lie algebra $\Sigma_{\mathbf{C}}^{0}$.

Next, we give properties on inhomogeneous Fourier integral operators $F(a, f, \tilde{\varphi})$ given in (1.6) as follows (cf. §7):

Proposition B. (i) There are a neighborhood $U$ of 1 of $\Sigma_{\mathbf{c}}^{0}$ and a neighborhood $W$ of the identity of $\mathscr{D}_{\omega}\left(S^{2 n-1}\right)$ such that if $F(a, f, \tilde{\varphi})=F\left(a^{\prime}, f^{\prime}, \tilde{\varphi}^{\prime}\right)$ for $a, a^{\prime} \in U, f, f^{\prime} \in H_{\mathbf{R}}^{1}$ and $\tilde{\varphi}, \tilde{\varphi}^{\prime} \in W$, then $a=a^{\prime}, f=f^{\prime}, \tilde{\varphi}=\tilde{\varphi}^{\prime}$ (cf. Lemma 5.1).

(ii) There are a neighborhood $U^{\prime}$ of 1 in $\Sigma_{\mathrm{c}}^{0}$ and a neighborhood $W^{\prime}$ of the identity of $\mathscr{D}_{\omega}\left(S^{2 n-1}\right)$ such that for any $(a, f, \tilde{\varphi}),(b, g, \tilde{\psi}) \in U^{\prime} \times H_{\mathbf{H}}^{1} \times W^{\prime}$, the composition $F(a, f, \tilde{\varphi}) \circ F(b, g, \tilde{\psi})$ is written in the shape $F(c, h, \tilde{\varphi} \circ \tilde{\psi})$ so that $c \in U, h \in H_{\mathbf{R}}^{1}, \tilde{\varphi} \circ \tilde{\psi} \in W$. Moreover, $c($ resp. $h)$ depends smoothly on $(a, b, f, g, \tilde{\varphi}, \tilde{\psi})($ resp. $(f, g, \tilde{\varphi}, \tilde{\psi}))$. In particular,

$$
F(a, f, \mathrm{id}) \circ F(b, g, \mathrm{id})=F(c, f+g, \mathrm{id}) \text {. }
$$

(iii) There are a neighborhood $U^{\prime \prime}$ of 1 in $\Sigma_{\mathbf{C}}^{0}$, a neighborhood $V^{\prime \prime}$ of 0 in $H_{\mathbf{R}}^{1}$ and a neighborhood $W^{\prime \prime}$ of the identity of $\mathscr{D}_{\omega}\left(S^{2 n-1}\right)$ such that for any $(a, f, \tilde{\varphi}) \in U^{\prime \prime} \times V^{\prime \prime} \times W^{\prime \prime}$, the operator $F(a, f, \tilde{\varphi})$ is invertible, and the inverse $F(a, f, \tilde{\varphi})^{-1}$ is written in the shape $F\left(c, h, \tilde{\varphi}^{-1}\right)$ so that $\left(c, h, \tilde{\varphi}^{-1}\right) \in U \times H_{\mathbf{R}}^{1} \times W$. Moreover, $c$ (resp. h) depends smoothly on $(a, f, \tilde{\varphi})(\operatorname{resp} .(f, \tilde{\varphi}))$.

Note that (i) in the above proposition shows that $(a, f, \tilde{\varphi})$ may be understood as the coordinate of the operator $F(a, f, \tilde{\varphi})$.

Set the group $G F^{\circ}$ as the group generated by

$$
\left\{F(a, f, \tilde{\varphi}) ;(a, f, \tilde{\varphi}) \in U^{\prime \prime} \times V^{\prime \prime} \times W^{\prime \prime}\right\} .
$$

Proposition B (ii), (iii) implies that the group operations are smooth on the neighborhood of identity. We easily see that the map (cf. Proposition B (i))

$$
\zeta: \mathscr{U} \rightarrow G F^{0} ; \quad(a, f, \tilde{\varphi}) \mapsto F(a, f, \tilde{\varphi}),
$$

where $\mathscr{U}=U^{\prime \prime} \oplus V^{\prime \prime} \oplus W^{\prime \prime}$, is an injective mapping satisfying the following conditions:

(1) $\zeta\left(1,0, \mathrm{id}_{\mathscr{D}_{\omega}\left(\mathbf{S}^{2 n-1}\right)}\right)=\mathrm{id}_{G F^{0}}$.

(2) For any $u_{1}, u_{2} \in \mathscr{U}$ such that $\zeta\left(u_{1}\right) \cdot \zeta\left(u_{2}\right) \in \operatorname{Im} \zeta$, there exist open neighborhoods $\mathscr{U}_{1}$ of $u_{1}$ in $\mathscr{U}$ and $\mathscr{U}_{2}$ of $u_{2}$ in $\mathscr{U}$ such that $\zeta^{-1}(\zeta() \cdot \zeta()): \mathscr{U}_{1} \times \mathscr{U}_{2} \rightarrow \mathscr{U}$ is well-defined and smooth.

(3) $\zeta^{-1}\left(\zeta()^{-1}\right): \mathscr{U} \rightarrow \mathscr{U}$ is smooth. 
Therefore, by the standard method, we can give the topology to $G F^{0}$ through the mapping $\zeta$. Since we can identify the neighborhood of $\operatorname{id}_{\mathscr{D}_{\omega}\left(S^{2 n-1}\right)}$ of $\mathscr{D}_{\omega}\left(S^{2 n-1}\right)$ with the neighborhood of $x \cdot \xi$ of $H_{\mathrm{R}}^{2}$ (cf. (5.40)), the tangent space at identity of $G F^{0}$ is $\sqrt{-1} H_{\mathbf{R}}^{2} \oplus \sqrt{-1} H_{\mathbf{R}}^{1} \oplus \Sigma_{\mathbf{C}}^{0}$. Thus, we obtain the following:

Proposition C. GF $F^{0}$ is a Fréchet-Lie group with the Lie algebra

$$
\sqrt{-1} H_{\mathbf{R}}^{2} \oplus \sqrt{-1} H_{\mathbf{R}}^{1} \oplus \Sigma_{\mathbf{C}}^{0} \text {. }
$$

By Proposition B (ii), (iii), $G \Sigma^{0}$ is a normal subgroup of $G F^{0}$, and obviously closed. For the group $G F^{0} / G \Sigma^{0}$, the coset $F\left(a, S_{1}, \tilde{\varphi}\right) \circ G \Sigma^{0}$ corresponds univalently to $\left(S_{\varphi}, S_{1}\right)$ by Proposition B (i). Therefore, $H_{\mathbf{R}}^{2} \oplus H_{\mathbf{R}}^{1}$ is the model space of $G F^{0} / G \Sigma^{0}$.

By Proposition B, we see that the map

$$
\tilde{\zeta}: \tilde{\mathscr{U}} \rightarrow G F^{0} / G \Sigma^{0} ; \quad\left(S_{\tilde{\varphi}}, S_{1}\right) \mapsto F\left(1, S_{1}, \tilde{\varphi}\right) \circ G \Sigma^{0},
$$

where $\tilde{\mathscr{U}}=W^{\prime \prime} \oplus V^{\prime \prime}$, is injective and satisfies the following conditions

(1) $\tilde{\zeta}\left(\operatorname{id}_{\mathscr{D} \omega}\left(S^{2 n-1}\right), 0\right)=e$.

(2) For any $u_{1}, u_{2} \in \tilde{\mathscr{U}}$ such that $\tilde{\zeta}\left(u_{1}\right) \cdot \tilde{\zeta}\left(u_{2}\right) \in \operatorname{Im} \tilde{\zeta}$, there exist open neighborhoods $\tilde{\mathscr{U}}_{1}$ of $u_{1}$ in $\tilde{\mathscr{U}}$ and $\tilde{\mathscr{U}}_{2}$ of $u_{2}$ in $\tilde{\mathscr{U}}$ such that $\tilde{\zeta}^{-1}(\tilde{\zeta}() \cdot \tilde{\zeta}()): \tilde{\mathscr{U}}_{1} \times \tilde{\mathscr{U}}_{2} \rightarrow \tilde{\mathscr{U}}$ is well-defined and smooth.

(3) $\tilde{\zeta}^{-1}\left(\tilde{\zeta}()^{-1}\right): \tilde{\mathscr{U}} \rightarrow \tilde{\mathscr{U}}$ is smooth.

Therefore $G F^{0} / G \Sigma^{0}$ is a Fréchet-Lie group.

By Proposition B (ii),

$$
F(a, f, \mathrm{id}) \circ F(b, g, \mathrm{id})=F(c, f+g, \mathrm{id}) .
$$

(3.24) means that $i_{c}: f \mapsto F(1, f$, id.) of (3.27) is well-defined as a homomorphism. On the other hand,

$$
\pi_{c}: f(a, f, \tilde{\varphi}) \mapsto \tilde{\varphi}
$$

gives a local homomorphism of $G F^{0} / G \Sigma^{0}$ into $\mathscr{D}_{\omega}\left(S^{2 n-1}\right)$ which covers a neighborhood of the identity of $\mathscr{D}_{\omega}\left(S^{2 n-1}\right)$. The kernel of $\pi_{c}$ is isomorphic to the space $H_{\mathrm{R}}^{1}$ of all homogeneous functions of degree 1 . Moreover, we see that

$$
j_{c}: \tilde{\varphi} \mapsto F(1,0, \tilde{\varphi})
$$

is a local section. Therefore we can consider the following local exact sequence:

$$
0 \longrightarrow H_{\mathbf{R}}^{1} \stackrel{i_{c}}{\longrightarrow} G F^{0} / G \Sigma^{0} \stackrel{\pi_{c}}{\longrightarrow} \mathscr{D}_{\omega}\left(S^{2 n-1}\right)_{0},
$$

where "local" means $\pi_{c}$ is a local homomorphism and $\operatorname{Im} i_{c}=\operatorname{Ker} \pi_{c}$. Here $H_{\mathbf{R}}^{1}$ is a regular Fréchet-Lie group, because it is a Fréchet space (cf. Remark after Definition 2.2).

As $\mathscr{D}_{\omega}\left(S^{2 n-1}\right)$ is a regular Fréchet-Lie group, the above local homomorphism combined with Lemmas 2.4 and 2.5 yields the following proposition: 
Proposition D. $G F^{0} / G \Sigma^{0}$ is a regular Fréchet-Lie group.

Once Proposition A and Proposition B are established, then the proof of Theorem A goes as follows: On the exact sequence

$$
1 \longrightarrow G \Sigma^{0} \stackrel{i}{\longrightarrow} G F^{0} \stackrel{\pi}{\longrightarrow} G F^{0} / G \Sigma^{0} \longrightarrow 1
$$

Proposition A shows that $G \Sigma^{0}$ is a regular Fréchet-Lie group. Proposition $\mathrm{C}$ shows that $G F^{0}$ is a Fréchet-Lie group. Proposition D shows that $G \Sigma^{0}$ is a closed normal subgroup of $G F^{0}$ with respect to the relative topology and $G F^{0} / G \Sigma^{0}$ is a regular Fréchet-Lie group. Furthermore, we now see that the mapping

$$
j: H_{\mathbf{R}}^{2} \oplus H_{\mathbf{R}}^{1} \ni\left(S_{2}, S_{1}\right) \mapsto F\left(1, S_{1}, \tilde{\varphi}_{S_{2}}\right) \in G F^{0}
$$

gives a local section, where $\tilde{\varphi}_{S_{2}}$ is a contact transformation on $S^{2 n-1}$. Therefore, by applying Lemma 2.5, we see that $G F^{0}$ is a regular Fréchet-Lie group. Theorem $\mathrm{A}$ is thereby proved.

Therefore, to complete the proof of Theorem A, we have only to prove Proposition A and Proposition B.

\section{Proof of Proposition A.}

In this section, we shall prove Proposition A in $\S 3$. Since the proof is fairly long, it will be splitted into several lemmas:

By the definition of $*$ in (3.19), it is easy to see that $*$ is a continuous bilinear mapping of $\Sigma_{\mathbf{C}}^{-i} \times \Sigma_{\mathbf{C}}^{-j}$ into $\Sigma_{\mathbf{C}}^{-(i+j)}(i, j \geq 0)$. However, to see the convergence of product integral, we need further the following property for the norms given in (3.6):

Proposition 4.1. For $m \geq 2 n+1$, the following inequality holds: for any $f, g \in \Sigma_{\mathbf{C}}^{-m}$,

$$
\llbracket f * g \rrbracket_{-M, k} \leq C_{k, M} \rrbracket f \rrbracket_{-M, k} \llbracket g \rrbracket_{-M, \delta_{k}} \quad(M \geq m),
$$

$C_{k, M}$, is a positive constant depending on $k, M$, and $\delta_{k}$ is a positive integer such that $\delta_{k} \geq k$ depending on $k$.

By a similar argument as in [OMYK]-V the above lemma yields the convergence of product integrals of $G \Sigma^{-m}$ for $m \geq 2 n+1$. To prove Proposition 4.1, we need the following lemma:

LEMMA 4.2. For any $r \in \mathfrak{B}_{\mathbf{C}}^{-M-1}, q \in \mathfrak{B}_{\mathbf{C}}^{-m}(m \geq 2 n+1), \theta \in[0,1]$ we have

$$
\begin{aligned}
& \langle x ; \xi\rangle^{M+1}\left|\iint r(x, \xi+\theta \eta) q(x+y, \xi) e^{-(i / \hbar) y \cdot \eta} d \eta \overline{d y}\right| \\
& \quad \leq C \sup \langle x ; \xi\rangle^{M+1}|r(x, \xi)| \sup _{|\lambda|+|\mu| \leq 2 l}\langle x ; \xi\rangle^{m}\left|\partial_{x}^{\lambda} \partial_{\xi}^{\mu} q(x, \xi)\right|,
\end{aligned}
$$


where $C$ is independent of $r, q, \theta$, and $l$ is any integer such that $2 l \geq M+n+1 \geq 3 n+$ $M-m+2$.

ProOF. First, we divide the domain of integration into two parts as follows:

$$
\left.I_{1}=\{(y, \eta) ;|\eta| \leq\langle\xi\rangle / 3\}, \quad I_{2}=\{(y, \eta) ;|\eta|\rangle\langle\xi\rangle / 3\right\} .
$$

On the set $I_{1}$, there exists $C(>0)$ such that

$$
C^{-1}\langle x ; \xi\rangle \leq\langle x ; \xi+\theta \eta\rangle \leq C\langle\dot{x} ; \xi\rangle, \quad \theta \in[0,1] .
$$

Using this, we obtain

$$
\begin{aligned}
& \left|\iint_{I_{1}} r(x, \xi+\theta \eta) q(x+y, \xi) e^{-(i / \hbar) y \cdot \eta} d y d \eta\right| \\
& \quad \leq \iint_{|\eta| \leq\langle\xi\rangle / 3} \frac{\sup _{\tilde{x}, \tilde{y}, \xi}\langle\tilde{x} ; \tilde{\xi}+\theta \tilde{\eta}\rangle^{M+1}|r(\tilde{x} ; \tilde{\xi}+\theta \tilde{\eta})|}{\langle x ; \xi\rangle^{M+1}} \times \frac{\sup _{\tilde{x}, \tilde{y}, \xi}\langle\tilde{x}+\tilde{y} ; \tilde{\xi}\rangle^{m}|q(\tilde{x}+\tilde{y} ; \tilde{\xi})|}{\langle x+y ; \xi\rangle^{m}} \tilde{d y} \tilde{d \eta} .
\end{aligned}
$$

As $m \geq 2 n+1$, the above quantity is bounded by

$$
C_{1} \frac{1}{\langle x ; \xi\rangle^{M+1}}\left\{\sup _{x, \xi}\langle x ; \xi\rangle^{M+1}|r(x, \xi)|\right\}\left\{\sup _{\substack{x, \xi \\|\lambda|+|\mu| \leq 2 l}}\langle x ; \xi\rangle^{m}\left|\partial_{x}^{\lambda} \partial_{\xi}^{\mu} q(x ; \xi)\right|\right\} .
$$

On $I_{2}$, as

$$
\langle x ; \xi+\theta \eta\rangle^{-M-1} \leq\langle x\rangle^{-M-1},
$$

and

$$
|\eta|^{-2 l}\left\{(\hbar / \sqrt{-1})^{2} \Delta_{y}\right\}^{l} e^{-(i / \hbar) y \cdot \eta}=e^{-(i / \hbar) y \cdot \eta}
$$

hold, we have the following estimate:

$$
\begin{aligned}
& \left|\iint_{I_{2}} r(x, \xi+\theta \eta) q(x+y, \xi) e^{-(i / \hbar) \eta y} d y d \eta\right| \\
& \leq \quad \iint_{|\eta|>\langle\xi\rangle / 3} \frac{1}{|\eta|^{2 l}} \frac{\sup _{\tilde{x}, \tilde{y}, \xi}\langle\tilde{x} ; \tilde{\xi}+\theta \tilde{\eta}\rangle^{M+1}|r(\tilde{x} ; \tilde{\xi}+\theta \tilde{\eta})|}{\langle x\rangle^{M+1}} \\
& \quad \times \frac{\sup _{\tilde{x}, \tilde{y}, \xi}\langle\tilde{x}+\tilde{y} ; \tilde{\xi}\rangle^{m}\left|\left\{(\hbar / \sqrt{-1})^{2} \Delta_{y}\right\}^{l} q(\tilde{x}+\tilde{y} ; \tilde{\xi})\right|}{\langle x+y ; \xi\rangle^{m}} d y \tilde{d \eta} .
\end{aligned}
$$

We choose $l$ such that $2 l>M+1+n$. By using $\langle x\rangle^{-M-1}\langle\xi\rangle^{-M-1} \leq\langle x ; \xi\rangle^{-M-1}$, the 
above quantity is bounded by

$$
C_{2} \frac{1}{\langle x ; \xi\rangle^{M+1}}\left\{\sup _{x, \xi}\langle x ; \xi\rangle^{M+1}|r(x, \xi)|\right\}\left\{\sup _{\substack{x, \xi \\|\lambda|+|\mu| \leq 2 l}}\langle x ; \xi\rangle^{m}\left|\partial_{x}^{\lambda} \partial_{\xi}^{\mu} q(x, \xi)\right|\right\} .
$$

By (4.2) and (4.3), we have the lemma by setting $C=\max \left\{C_{1}, C_{2}\right\}$.

Proof of Proposition 4.1. For any $f \in \Sigma_{\mathrm{c}}^{-m}$, we write as

$$
f=\sum_{j=m}^{M} \pi_{-j}(f)+r_{-M}(f)
$$

where $\pi_{-j}(f)=f_{-j} \rho^{-j}$ outside some compact subset.

By the bilinearity of (3.19), we have

$$
f * g=\sum_{i, j} \pi_{-i}(f) * \pi_{-j}(g)+\sum_{j} r_{-M}(f) * \pi_{-j}(g)+\sum_{i} \pi_{-i}(f) * r_{-M}(g)+r_{-M}(f) * r_{-M}(g) .
$$

To show Proposition 4.1, it is enough to see that

(i) $\llbracket \pi_{-i}(f) * \pi_{-j}(g) \rrbracket_{-M, k} \leq C \rrbracket \pi_{-i}(f) \rrbracket_{-M, k} \rrbracket \pi_{-j}(g) \rrbracket_{-M, \delta_{k}}$,

(ii) $\left\|r_{-M}(f) * \pi_{-j}(g)\right\|_{-M-1, k-M-1} \leq C\left\|\mid r_{-M}(f)\right\|_{-M-1, k-M-1} \rrbracket \pi_{-j}(g) \|_{-M, \delta_{k}}$,

(iii) $\left\|\pi_{-i}(f) * r_{-M}(g)\right\|_{-M-1, k-M-1} \leq C \rrbracket \pi_{-i}(f) \rrbracket_{-M, k}\left\|r_{-M}(g)\right\|_{-M-1, \delta_{k}-M-1}$,

(iv) $\left\|r_{-M}(f) * r_{-M}(g)\right\|_{-M-1, k-M-1} \leq C\left\|r_{-M}(f)\right\|_{-M-1, k-M-1}\left\|r_{-M}(g)\right\|_{-M-1, k-M-1}$.

By applying (3.19) to the inside of the left hand side of (ii) and (iv) of (4.5), we have

$$
\begin{aligned}
& r_{-M}(f) * \pi_{-j}(g)=\left.\left.\iint r_{-M}(f)\right|_{(x, \xi+\eta)} \pi_{-j}(g)\right|_{(x+y, \xi)} e^{(i / \hbar) y \cdot \eta} \overline{d y} \overline{d \eta}, \\
& r_{-M}(f) * r_{-M}(g)=\left.\left.\iint r_{-M}(f)\right|_{(x, \xi+\eta)} r_{-M}(g)\right|_{(x+y, \xi)} e^{(i / \hbar) y \cdot \eta} \not{d y d \eta} .
\end{aligned}
$$

Applying Lemma 4.2, (ii), (iv) holds by setting $\delta_{k}=k+2 l$.

Next, we shall show (i), (iii). By applying (3.20), we have the following:

$$
\pi_{-i}(f) * \pi_{-j}(g)=\sum_{\substack { l=m \\
\begin{subarray}{c}{i+j=l-2|\alpha| \\
m \leq i, j \leq M{ l = m \\
\begin{subarray} { c } { i + j = l - 2 | \alpha | \\
m \leq i , j \leq M } }\end{subarray}} \frac{1}{\alpha !} \not \not_{\xi}^{\alpha} \pi_{-i}(f) \partial_{x}^{\alpha} \pi_{-j}(g)+r_{-M}\left(\pi_{-i}(f) * \pi_{-j}(g)\right)
$$

where

$$
\begin{aligned}
& r_{-M}\left(\pi_{-i}(f) * \pi_{-j}(g)\right)(x, \xi) \\
& \quad=\left.\left.\iiint_{0}^{1} \frac{M+1}{\alpha !}(1-\theta)^{M} \not D_{\xi}^{\alpha} \pi_{-i}(f)\right|_{(x, \xi+\theta \eta)} \partial_{x}^{\alpha} \pi_{-j}(g)\right|_{(x+y, \xi)} d \theta e^{(i / \hbar) y \cdot \eta} \not y d \eta .
\end{aligned}
$$

Since the absolute value of the factor of homogeneous degree 0 of the derivative of $\partial_{\xi}^{\alpha}\left(f_{-i} r^{-i}\right) \partial_{x}^{\alpha}\left(g_{-j} r^{-j}\right)$ of order $k-(i+j+2|\alpha|)$ is bounded up to constant factor by 
$\llbracket \pi_{-i}(f) \rrbracket_{k} \cdot \llbracket \pi_{-i}(g) \rrbracket_{k}$, it is easy to estimate the first term of the right hand side of (4.7). Thus, it is enough to estimate (4.8). Using the Taylor's theorem, integration by part and the Fourier inversion formula, we see

$$
\begin{aligned}
& \left.\quad \partial_{x}^{\varepsilon} \partial_{\xi}^{\delta_{\xi} r_{-M}(\pi-i}(f) * \pi_{-j}(g)\right)(x, \xi) \\
& =\sum_{\substack{\varepsilon_{1}+\varepsilon_{2}=\varepsilon \\
\delta_{1}+\delta_{2}=\delta}} \frac{\varepsilon !}{\varepsilon_{1} ! \varepsilon_{2} !} \frac{\delta !}{\delta_{1} ! \delta_{2} !}\left[\sum_{i+j+2|\alpha|=M+1} \int_{0}^{1} \frac{M+1}{\alpha !}(1-\theta)^{M}\right. \\
& \quad \times\left.\sum_{|\gamma|+|\alpha|+\left|\varepsilon_{1}\right|+\left|\delta_{1}\right|+i \leq M} \frac{1}{\gamma !} \theta^{|\gamma| \partial_{x}^{\varepsilon_{1}}} \partial_{\xi}^{\delta_{1}} \not D_{\xi}^{\alpha} \not D_{\xi}^{\gamma} \pi_{-i}(f) \partial_{x}^{\varepsilon_{2}} \partial_{\xi}^{\delta_{2}} \partial_{x}^{\alpha} \partial_{x}^{\gamma} \pi_{-j}(g) d \theta\right|_{(x, \xi)} \\
& +\sum_{\substack{i+j+2|\alpha| \\
=}} \sum_{\substack{|\gamma+1 \\
+| \delta_{1} \mid+i=M+1}} \iint\left\{\int_{0}^{1} \int_{0}^{1} \frac{M+1}{\alpha !}(1-\theta)^{M} \frac{\Lambda\left(\varepsilon_{1}, \delta_{1}\right)}{\gamma !} \theta^{|\gamma|}(1-\tau)^{\Lambda\left(\varepsilon_{1}, \delta_{1}\right)-1}\right. \\
& \left.\left.\quad \times\left.\left.\partial_{x}^{\varepsilon_{1}} \partial_{\xi}^{\delta_{1}} \not D_{\xi}^{\alpha} \not D_{\xi}^{\gamma} \pi_{-i}(f)\right|_{(x, \xi+\theta \tau \eta)} \partial_{x}^{\varepsilon_{2}} \partial_{\xi}^{\delta_{2}} \partial_{x}^{\alpha} \partial_{x}^{\gamma} \pi_{-j}(g)\right|_{(x+y, \xi)} e^{-(i / \hbar) y \cdot \eta} d \tau d \theta\right\} d y d \eta\right],
\end{aligned}
$$

where $\Lambda\left(\varepsilon_{1}, \delta_{1}\right)=M+1-i-|\alpha|-\left|\varepsilon_{1}\right|-\left|\delta_{1}\right|$.

Note that only $\partial_{x}^{\varepsilon_{1}} \partial_{\xi}^{\delta_{1}} \not D_{\xi}^{\alpha} \not D_{\xi}^{\gamma} \pi_{-i}(f)$ such that

$$
|\gamma|+|\alpha|+\left|\varepsilon_{1}\right|+\left|\delta_{1}\right|+i \leq M+1+|\varepsilon|+|\delta|
$$

appears in (4.9). Then applying Lemma 4.2, we see that (i) holds by setting $\delta_{k}=k+2 l$ $(k=M+1+|\varepsilon|+|\delta|)$.

As for (iii), we have by the same manner that

$$
\begin{aligned}
& \pi_{-i}(f) * r_{-M}(g)(x, \xi)=\left.\sum_{|\gamma|+i \leq M} \frac{1}{\gamma !} \not D_{\xi}^{\gamma} \pi_{-i}(f) \partial_{x}^{\gamma} r_{-M}(g)\right|_{(x, \xi)}
\end{aligned}
$$

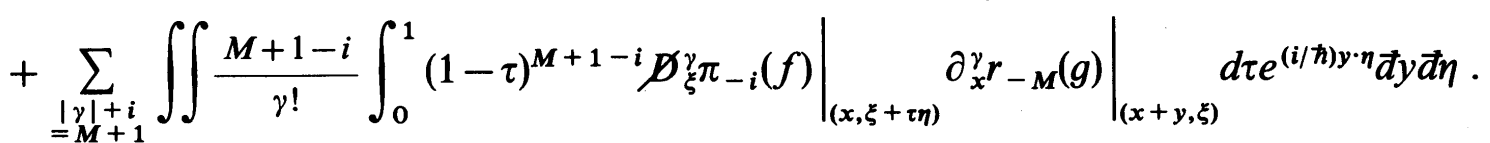

Therefore by Lemma 4.2, we see (iii) holds.

Next, we shall prove the following:

Proposition 4.3. $G \Sigma^{-m}$ is an open subset in $1+\Sigma_{\mathbf{C}}^{-m}$.

At first, we set a positive constant $\varepsilon_{1}$ by

$$
\varepsilon_{1}=\left(2 \int\langle x\rangle^{-n-1} d x \int\langle\xi\rangle^{-n-1} \bar{d} \xi+1\right)^{-1} .
$$

Denote by $\mathscr{S}\left(\mathbf{R}^{2 n}\right)$ the space of rapidly decreasing functions on $\mathbf{R}^{2 n}$.

LEMMA 4.4. Let $k(x, \xi) \in \mathscr{S}\left(\mathbf{R}^{2 n}\right)$. If $k(x, \xi)$ satisfies $\left|\langle x ; \xi\rangle^{2 n+2} k(x, \xi)\right| \leq \varepsilon_{1}$, then there exists $h \in \mathscr{S}\left(\mathbf{R}^{2 n}\right)$ such that 


$$
(1-P(k)) \circ(1+P(h))=(1+P(h)) \circ(1-P(k))=\mathrm{Id} .
$$

Proof. By (3.19), we have that the product $k^{* l}=\overbrace{k * \cdots * k}^{l \text { times }}$ is given as follows: using the convention $y_{0}=0, \eta_{l}=0$,

$$
\begin{array}{r}
k^{* l}=\iint \cdots \iint \prod_{i=0}^{l-1} k\left(x+y_{i}, \xi+\eta_{i+1}\right) e^{-(i / \hbar)\left\{\sum_{i=1}^{l-1} \eta_{i} \cdot y_{i}-\sum_{i=1}^{l-2} y_{i} \cdot \eta_{i+2}\right\}} \\
\times \not y_{1} \not \eta_{1} \cdots d y_{l-1} \not \eta_{l-1} .
\end{array}
$$

By the assumption of Lemma 4.4, we obtain

$$
\left|k^{* l}(x, \xi)\right| \leq \prod_{i=0}^{l-1} \frac{\varepsilon_{1}}{\left\langle x+y_{i} ; \xi+\eta_{i+1}\right\rangle} \leq\left(\frac{1}{2}\right)^{l}\langle x ; \xi\rangle .
$$

Thus, $\sum_{l=0}^{\infty} k^{* l}(x, \xi)$ is well-defined as a $C^{0}$-function on $\mathbf{R}^{2 n}$.

A direct computation gives

$$
\begin{aligned}
&\langle x ; \xi\rangle^{N}\left|\sum_{l=0}^{\infty} \partial_{x}^{\alpha} \partial_{\xi}^{\beta} k^{* l}\langle x, \xi\rangle\right| \\
& \leq\langle x\rangle^{N}\langle\xi\rangle^{N} \mid \sum_{l=0}^{\infty} \iint \cdots \iint_{\substack{\alpha_{(1)}+\cdots+\alpha_{(l)}=\alpha \\
\beta_{(1)}+\cdots+\beta_{(l)}=\beta}} \frac{\alpha !}{\alpha_{(1)} ! \cdots \alpha_{(l)} !} \frac{\beta !}{\beta_{(1)} ! \cdots \beta_{(l)} !} \\
& \quad \times \prod_{i=0}^{l-1} \partial_{x}^{\alpha_{(i+1)}} \partial_{\xi}^{\beta_{(i+1)}} k\left(x+y_{i}, \xi+\eta_{i+1}\right) d y_{1} d \eta_{1} \cdots d y_{l-1} d \eta_{l-1} \mid .
\end{aligned}
$$

Denote the right hand side of (4.14) by $J$. If $|\alpha+\beta| \leq l$ in the above inequality (4.14), then there are $l-|\alpha+\beta|$ factors which are not differentiated. Other factors are differentiated at most $|\alpha+\beta|$-times. Since

$$
\begin{aligned}
\left|\partial_{x}^{\alpha_{(1)}} \partial_{\xi}^{\beta_{(1)}} k\left(x, \xi+\eta_{1}\right)\right| \leq & C_{\alpha_{(1)}, \beta_{(1)}, N+2 n+2}\left\langle x ; \xi+\eta_{1}\right\rangle^{-N-2 n-2} \\
& \times\left|\left\langle x ; \xi+\eta_{1}\right\rangle^{N+2 n+2} \partial_{x}^{\alpha_{(1)}} \partial_{\xi(1)}^{\beta_{(1)}} k\left(x, \xi+\eta_{1}\right)\right| \\
\leq & C\langle x\rangle^{-N}\left\langle\xi+\eta_{1}\right\rangle^{-2 n-2}, \\
\left|\partial_{x}^{\alpha_{(l)}} \partial_{\xi}^{\beta_{(l)}} k\left(x+y_{l-1}, \xi\right)\right| \leq & C\langle\xi\rangle^{-N}\left\langle x+y_{l-1}\right\rangle^{-2 n-2},
\end{aligned}
$$

the factor $\langle x\rangle^{N}\langle\xi\rangle^{N}$ is cancelled out. So, we get

$$
\begin{aligned}
J & \leq\left|\sum_{l=0}^{\infty}\left(C+\frac{1}{2}\right)^{|\alpha+\beta|}\left(\frac{1}{2}\right)^{l-|\alpha+\beta|} \times \sum_{\substack{\alpha_{(1)}+\cdots+\alpha_{(l)}=\alpha \\
\beta_{(1)}+\cdots+\beta(l)=\beta}} \frac{\alpha !}{\alpha_{(1)} ! \cdots \alpha_{(l)} !} \frac{\beta !}{\beta_{(1)} ! \cdots \beta_{(l)} !}\right| \\
& \leq \sum_{l=0}^{\infty}\left(C+\frac{1}{2}\right)^{|\alpha+\beta|}\left(\frac{1}{2}\right)^{l-|\alpha+\beta|} l^{|\alpha+\beta|}<\infty \quad(\forall N, \forall \alpha, \forall \beta) .
\end{aligned}
$$


Thus, we have $\sum_{l=0}^{\infty} k^{* l} \in \mathscr{S}\left(\mathbf{R}^{2 n}\right)$. The desired $h$ is given by $\sum_{l=1}^{\infty} k^{* l}$.

Proof of Proposition 4.3. First, we fix $m \geq 0$. For any $f \in \Sigma_{\mathbf{c}}^{-m}$, let

$$
f \sim f_{-m} \rho^{-m}+f_{-m-1} \rho^{-m-1}+f_{-m-2} \rho^{-m-2}+\cdots, \quad f_{-j} \in C_{\mathbf{C}}^{\infty}\left(S^{2 n-1}\right)
$$

be the asymptotic expansion of $f$. Using $\left\{f_{-j}\right\}_{j \geq m}$, we set the smooth functions of $S^{2 n-1}$ :

$$
\begin{aligned}
& g_{-m-k}=-f_{-m-k}, \quad 0 \leq k \leq m-1, \\
& g_{-2 m-l}=-f_{-2 m-l}-\sum_{\substack{2|\alpha|+i+j=2 m+l \\
i, j<2 m+l}} \frac{1}{\alpha !} \not D_{\xi}^{\alpha}\left(f_{-i} \rho^{-j}\right) \partial_{x}^{\alpha}\left(g_{-j} \rho^{-j}\right) \rho^{2 m+l}, \quad l \geq 0 .
\end{aligned}
$$

By Lemma 3.3, for any $M \geq m$ there exists $g \in \Sigma_{\mathbf{C}}^{-m}$ satisfying (3.10) and (3.11). Setting $s_{f}=(1+f) *(1+g)-1$, we see that $s_{f} \in \mathscr{S}\left(\mathbf{R}^{2 n}\right)$ and

$$
\begin{aligned}
s_{f}= & (1+f) * r_{-M-1}(g)+\left(\sum_{i=m}^{M} \pi_{-i}(f)\right) *\left(\sum_{j=m}^{M} \pi_{-j}(g)\right) \\
& +r_{-M-1}(f)+r_{-M-1}(f) *\left(\sum_{j=m}^{M} \pi_{-j}(g)\right) .
\end{aligned}
$$

Since $\delta(>0)$ in (3.11) can be chosen very small, if $f$ is sufficiently close to 0 , then $s_{f}$ is small so that the assumption of Lemma 4.4 is satisfied. By Lemma 4.4 , there exists $h_{f} \in \mathscr{S}\left(\mathbf{R}^{2 n}\right)$ such that $\left(1+s_{f}\right) *\left(1+h_{f}\right)=1$. Hence, $(1+g) *\left(1+h_{f}\right)$ is the inverse of $1+f$. It follows that $G \Sigma^{-m}$ contains a neighborhood of 1 of $1+\Sigma_{\mathbf{C}}^{-m}$. By using the continuity of the product $*, G \Sigma^{-m}$ is an open subset of $1+\Sigma_{\mathbf{C}}^{-m}$.

By the above lemma, we see that $G \Sigma^{-m}$ is a Fréchet-Lie group under the relative topology in $1+\Sigma_{\mathbf{C}}^{-m}$. By Proposition 4.1, the product integral is well-defined on $G \Sigma^{-m}$. Therefore, we have the following:

LEMMA 4.5. $G \Sigma^{-m}$ is a regular Fréchet-Lie group for any $m \geq 2 n+1$.

Note that, $\Sigma_{\mathbf{C}}^{-m}$ is a closed subset of $\Sigma_{\mathbf{C}}^{-l}$ for any $m, l$ such that $m \geq l$ and $G \Sigma^{-l} \cap \Sigma_{\mathbf{C}}^{-m}=$ $G \Sigma^{-m}$. Thus, we have the following:

LEMMA 4.6. $G \Sigma^{-m}$ is a closed normal subgroup of $G \Sigma^{-l}(m \geq l)$.

We have already shown that $G \Sigma^{0}$ can be identified with an open subset of $\Sigma_{\mathbf{C}}^{\mathbf{0}}$ (cf. Proposition 4.3) then it follows that $G \Sigma^{0}$ forms a topological group under the relative topology in $\Sigma_{\mathbf{C}}^{\mathbf{0}}$. Note that this topology is much stronger than the uniform topology for operators. We have seen also that $G \Sigma^{-m}$ are closed normal subgroups of $G \Sigma^{0}$ for any $m=0,1,2, \cdots$ (cf. Lemma 4.6). By using these subgroups, we consider the following exact sequences: 


$$
1 \longrightarrow G \Sigma^{-1} \stackrel{i_{\Sigma}}{\longrightarrow} G \Sigma^{0} \stackrel{\pi_{\Sigma}}{\longrightarrow} H_{\mathbf{C}, *}^{0} \longrightarrow 1
$$

$$
1 \longrightarrow G \Sigma^{-i} \stackrel{i_{\Sigma}}{\longrightarrow} G \Sigma^{-i+1} \stackrel{\pi_{\Sigma}}{\longrightarrow} H_{\mathbf{C}}^{-i+1} \longrightarrow 0, \quad(i \geq 2)
$$

where $H_{\mathbf{C}, *}^{\mathbf{0}}$ is a multiplicative group of all $\mathbf{C}-\{0\}$ valued smooth functions of homogeneous degree 0 which is a regular Fréchet-Lie group.

At first, we can easily see that there exist local sections $j_{\Sigma}: f \mapsto P(1+\kappa f)$ of the second exact sequence of (4.17). Since $G \Sigma^{-m}$ is a closed normal regular Fréchet-Lie group for $m \geq 2 n+1$ under the relative topology (cf. Lemma 4.5) and $H_{\mathbf{C}}^{-m+1}$ is an abelian regular Fréchet-Lie group, Lemma 2.5 shows that $G \Sigma^{-m+1}$ is also a regular Fréchet-Lie group. We can repeat this procedure and hence we get $G \Sigma^{-1}$ is a regular Fréchet-Lie group.

On the first exact sequence of (4.17), the mapping

$$
j_{\Sigma}: f \mapsto P(1+\kappa(f-1))
$$

defined for $f$ which is sufficiently close to 1 of $H_{\mathbf{c}, *}^{0}$ gives local section. Thus, by Lemma 2.5, we have the following corollary which includes Proposition A:

Corollary 4.7. $G \Sigma^{0}, \cdots, G \Sigma^{-m+1}, G \Sigma^{-m}, \cdots$ are regular Fréchet-Lie groups.

\section{Canonical transformations and generating functions.}

In this section we shall investigate relations of canonical transformation with its generating function.

It is well-known in symplectic geometry that a canonical transformation which is close to the identity corresponds bijectively to a function close to $\bar{x} \cdot \xi$, which is called the generating function. This correspondence is given through another transformation, which will be called a cross mapping in this paper. We start with analyzing this correspondence.

Any $C^{\infty}$ function $S$ gives a mapping $\chi_{s}$ defined by

$$
\chi_{s}: \mathbf{R}_{(\bar{x}, \xi)}^{2 n} \rightarrow \mathbf{R}_{(x, \bar{\xi})}^{2 n} ; \quad \chi_{s}\left(\begin{array}{c}
\bar{x} \\
\xi
\end{array}\right)=\left(\begin{array}{c}
\nabla_{\xi} S(\bar{x}, \xi) \\
\nabla_{\bar{x}} S(\bar{x}, \xi)
\end{array}\right) .
$$

We shall call $\chi_{s}$ the cross mapping defined by $S$.

The following lemma is rather well-known (See [S] for the proof):

LEMMA 5.1 (global implicit function theorem). Let

$$
\Psi: \mathbf{R}^{l} \rightarrow \mathbf{R}^{l}
$$

be a $C^{1}$ mapping. If there exists $K>0$ such that 


$$
K^{-1} \leq\left|\operatorname{det} \partial_{x} \Psi(x)\right|, \quad\left\|\partial_{x} \Psi(x)\right\| \leq K \quad\left(\forall x \in \mathbf{R}^{l}\right),
$$

then $\Psi$ is $C^{1}$ diffeomorphism. Here $\|\cdot\|$ is uniform norm of matrix.

If $S$ is sufficiently close to $\bar{x} \cdot \xi$ in the certain topology, then Lemma 5.1 shows that the following transformation $\varphi_{S}$ is well-defined as a global diffeomorphism on $\boldsymbol{R}^{2 n}$ :

$$
\begin{aligned}
\varphi_{S}: \begin{array}{c}
\mathbf{R}_{(x, \xi)}^{2 n} \\
w
\end{array} & \left.\rightarrow \begin{array}{c}
\mathbf{R}_{(\bar{x}, \bar{\xi})}^{2 n} \\
\omega \\
\left(\begin{array}{c}
\nabla_{\xi} S(\bar{x}, \xi) \\
\xi
\end{array}\right)
\end{array}\right) \mapsto\left(\begin{array}{c}
\bar{x} \\
\nabla_{\bar{x}} S(\bar{x}, \xi)
\end{array}\right) .
\end{aligned}
$$

Since $d \bar{x} \wedge d \bar{\xi}=d \bar{x} \wedge\left(\nabla_{\xi} \nabla_{\bar{x}} S\right) d \xi=\left(\nabla_{\bar{x}} \nabla_{\xi} S\right) d \bar{x} \wedge d \xi=d x \wedge d \xi$, we see that $\varphi_{S}$ is a canonical transformation. Such a $\varphi_{S}$ is called the canonical transformation generated by $S$, and $S$ is called the generating function of $\varphi_{S}$.

Note that the relation between the cross mapping $\chi_{s}$ and the canonical transformation $\varphi_{S}$ is given by

$$
\left(\begin{array}{l}
x \\
\bar{\xi}
\end{array}\right)=\chi_{S}\left(\begin{array}{l}
\bar{x} \\
\xi
\end{array}\right) \text { if and only if }\left(\begin{array}{l}
\bar{x} \\
\bar{\xi}
\end{array}\right)=\varphi_{S}\left(\begin{array}{l}
x \\
\xi
\end{array}\right) .
$$

The graph of cross mapping $\chi_{s}$ coincides with the graph of $\varphi_{S}$ in the space $\mathbf{R}^{4 n}$. We denote $\chi_{s}$ also by $\chi_{\varphi_{S}}$.

Conversely, the following is easy to show:

LEMMA 5.2. If $\varphi\left(\begin{array}{l}x \\ \xi\end{array}\right)=\left(\begin{array}{l}\bar{x} \\ \bar{\xi}\end{array}\right)$ can be solved with respect to $\left(\begin{array}{l}x \\ \bar{\xi}\end{array}\right)$, i.e.

$$
\chi\left(\begin{array}{l}
\bar{x} \\
\xi
\end{array}\right)=\left(\begin{array}{l}
x \\
\bar{\xi}
\end{array}\right)=\left(\begin{array}{l}
x(\bar{x}, \xi) \\
\bar{\xi}(\bar{x}, \xi)
\end{array}\right),
$$

then

$$
\bar{\xi}(\bar{x}, \xi) \cdot d \bar{x}+x(\bar{x}, \xi) \cdot d \xi
$$

is a closed form, and $\chi$ is a cross mapping defined by

$$
S(\bar{x}, \xi)=\int_{0}^{1}\{\bar{\xi}(t \bar{x}, t \xi) \cdot \bar{x}+x(t \bar{x}, t \xi) \cdot \xi\} d t .
$$

As the generating function $S$ is uniquely determined up to additive constants by a canonical transformation $\varphi$ to which Lemma 5.1 can be applied, we shall denote this by $\sigma_{\varphi}$, i.e. $S=\sigma_{\varphi}$.

Given generating functions $S, T$, we compute the generating function $\sigma_{\varphi_{S} \circ \varphi_{T}}$ of the composition for canonical transformations $\varphi_{S}, \varphi_{T}$.

By (5.5), we have 


$$
\begin{aligned}
& \left(\begin{array}{c}
\bar{x}_{1} \\
\bar{\xi}
\end{array}\right)=\chi_{S}\left(\begin{array}{c}
\overline{\bar{x}} \\
\bar{\xi}_{1}
\end{array}\right)=\left(\begin{array}{c}
\nabla_{\bar{\xi}_{1}} S\left(\bar{x}, \bar{\xi}_{1}\right) \\
\nabla_{\bar{x}} S\left(\bar{x}, \bar{\xi}_{1}\right)
\end{array}\right) \text { iff }\left(\begin{array}{c}
\overline{\bar{x}} \\
\bar{\xi}
\end{array}\right)=\varphi_{S}\left(\begin{array}{c}
\bar{x}_{1} \\
\bar{\xi}_{1}
\end{array}\right), \\
& \left(\begin{array}{c}
x \\
\bar{\xi}_{2}
\end{array}\right)=\chi_{T}\left(\begin{array}{c}
\bar{x}_{2} \\
\xi
\end{array}\right)=\left(\begin{array}{c}
\nabla_{\xi} T\left(\bar{x}_{2}, \xi\right) \\
\nabla_{\bar{x}_{2}} T\left(\bar{x}_{2}, \xi\right)
\end{array}\right) \text { iff }\left(\begin{array}{c}
\bar{x}_{2} \\
\bar{\xi}_{2}
\end{array}\right)=\varphi_{T}\left(\begin{array}{c}
x \\
\xi
\end{array}\right) .
\end{aligned}
$$

The graph of the cross mapping $\chi_{\varphi_{S} \circ \varphi_{T}}$ expresses the relation of $\left(\begin{array}{l}\bar{x} \\ \xi\end{array}\right)$ and $\left(\begin{array}{l}x \\ \bar{\xi}\end{array}\right)$ such that

$$
\left(\begin{array}{l}
\bar{x}_{1} \\
\bar{\xi}_{1}
\end{array}\right)=\left(\begin{array}{c}
\bar{x}_{2} \\
\bar{\xi}_{2}
\end{array}\right), \quad\left(\begin{array}{c}
\bar{x}_{1} \\
\bar{\xi}
\end{array}\right)=\chi_{S}\left(\begin{array}{l}
\bar{x}_{\xi} \\
\bar{\xi}_{1}
\end{array}\right), \quad\left(\begin{array}{c}
x \\
\bar{\xi}_{2}
\end{array}\right)=\chi_{T}\left(\begin{array}{c}
\bar{x}_{2} \\
\xi
\end{array}\right) .
$$

Set $\bar{\xi}_{1}=\bar{\xi}_{2}, \bar{x}_{1}=\bar{x}_{2}$, and solve the equations

$$
\left\{\begin{array}{l}
\bar{x}-\nabla_{\bar{\xi}} S(\bar{x}, \bar{\xi})=0 \\
\bar{\xi}-\nabla_{\bar{x}} T(\bar{x}, \xi)=0
\end{array}\right.
$$

where we put $\bar{\xi}=\bar{\xi}_{1}=\bar{\xi}_{2}, \bar{x}=\bar{x}_{1}=\bar{x}_{2}$. Then, we obtain the cross mapping $\chi_{\varphi_{S} \circ \varphi_{T}}$.

By using Lemma 5.1, we can solve the equation (5.7) globally on $\mathbf{R}^{2 n}$ as

$$
\bar{x}=\bar{x}(\bar{x}, \xi), \quad \bar{\xi}=\bar{\xi}(\bar{x}, \xi) .
$$

Substituting (5.8) to (5.6), we have

$$
\begin{aligned}
& \chi_{S}\left(\begin{array}{c}
\bar{x} \\
\bar{\xi}(\bar{x}, \xi)
\end{array}\right)=\left(\begin{array}{c}
\nabla_{\bar{\xi}} S(\bar{x}, \bar{\xi}(\bar{x}, \xi)) \\
\nabla_{\bar{x}} S(\bar{x}, \bar{\xi}(\bar{x}, \xi))
\end{array}\right)=\left(\begin{array}{c}
\bar{x}(\bar{x}, \xi) \\
\bar{\xi}
\end{array}\right), \\
& \chi_{T}\left(\begin{array}{c}
\bar{x}(\bar{x}, \xi) \\
\xi
\end{array}\right)=\left(\begin{array}{c}
\nabla_{\xi} T(\bar{x}(\bar{x}, \xi), \xi) \\
\nabla_{\bar{x}} T(\bar{x}(\bar{x}, \xi), \xi)
\end{array}\right)=\left(\begin{array}{c}
x \\
\bar{\xi}(\bar{x}, \xi)
\end{array}\right) .
\end{aligned}
$$

Since $\chi_{\varphi_{S} \circ \varphi_{T}}\left(\begin{array}{c}\bar{x} \\ \xi\end{array}\right)=\left(\begin{array}{l}x \\ \bar{\xi}\end{array}\right)$, we have

$$
\chi_{\varphi_{S} \circ \varphi_{T}}\left(\begin{array}{c}
\bar{x} \\
\xi
\end{array}\right)=\left(\begin{array}{l}
\left(\nabla_{\xi} T\right)(\bar{x}(\bar{x}, \xi), \xi) \\
\left(\nabla_{\bar{x}} S\right)(\bar{x}, \bar{\xi}(\bar{x}, \xi))
\end{array}\right) .
$$

LEMMA 5.3. The generating function $\sigma_{\varphi_{S} \circ \varphi_{T}}$ is given by

$$
S(\bar{x}, \bar{\xi}(\bar{x}, \xi))-\langle\bar{\xi}(\bar{x}, \xi) \mid \bar{x}(\bar{x}, \xi)\rangle+T(\bar{x}(\bar{x}, \xi), \xi),
$$

where $\langle\cdot \mid \cdot\rangle$ means the usual inner product on $\mathbf{R}^{n}$.

For simplicity of notations, we shall denote (5.11) by $S \odot T$.

Proof. The cross mapping $\chi_{S \odot T}\left(\begin{array}{l}\bar{x} \\ \xi\end{array}\right)=\left(\begin{array}{l}x \\ \bar{\xi}\end{array}\right)$ is given by

$$
\left(\begin{array}{l}
x \\
\bar{\xi}
\end{array}\right)=\left(\begin{array}{l}
\nabla_{\bar{\xi}}(S \odot T)(\bar{x}, \xi) \\
\nabla_{\bar{x}}(S \odot T)(\bar{x}, \xi)
\end{array}\right) .
$$


By using (5.7) and (5.8), we have

$$
\begin{aligned}
\nabla_{\xi}(S \odot T)(\bar{x}, \xi)= & \left(\nabla_{\bar{\xi}} S\right)(\bar{x}, \bar{\xi}(\bar{x}, \xi)) \cdot \nabla_{\xi} \bar{\xi}(\bar{x}, \xi) \\
& -\nabla_{\xi} \bar{\xi}(\bar{x}, \xi) \cdot \bar{x}(\bar{x}, \xi)-\bar{\xi}(\bar{x}, \xi) \cdot \nabla_{\xi} \bar{x}(\bar{x}, \xi) \\
& +\left(\nabla_{\bar{x}} T\right)(\bar{x}(\bar{x}, \xi), \xi) \cdot \nabla_{\bar{\xi}} \bar{x}(\bar{x}, \xi)+\left(\nabla_{\xi} T\right)(\bar{x}(\bar{x}, \xi), \xi) \\
= & \left(\nabla_{\xi} T\right)(\bar{x}(\bar{x}, \xi), \xi) .
\end{aligned}
$$

Similarly, we see that

$$
\nabla_{\bar{x}}(S \odot T)(\bar{x}, \xi)=\left(\nabla_{\bar{x}} S\right)(\bar{x}, \xi(\bar{x}, \xi)) .
$$

Therefore we see, by (5.10), that

$$
\chi_{S \odot T}\left(\begin{array}{l}
\bar{x} \\
\xi
\end{array}\right)=\chi_{\varphi_{S} \circ \varphi_{T}}\left(\begin{array}{l}
\bar{x} \\
\xi
\end{array}\right) .
$$

It follows that ${\sigma_{\varphi_{S} \circ \varphi_{T}}}=S \odot T$ up to additional constants.

$S \odot T$ will be called the composed generating function of $S$ and $T$.

Now, suppose $S$ and $T$ are elements of $H_{\mathrm{R}}^{2} \oplus H_{\mathrm{R}}^{1}$ which are sufficiently close to $x \cdot \xi$. Although these functions are not necessarily defined on the whole space $\mathbf{R}^{2 n}$, we can apply Lemma 5.3 to functions

$$
S_{\kappa}=\kappa S+(1-\kappa) x \cdot \xi, \quad T_{\kappa}=\kappa T+(1-\kappa) x \cdot \xi
$$

instead of $S, T$, where $\kappa$ is given by (3.2). Note that if $S=S_{2}+S_{1} \in H_{\mathbf{R}}^{2} \oplus H_{\mathrm{R}}^{1}$, then $S_{\kappa}=\kappa S_{2}+\kappa S_{1}+S_{(0,-\infty)} \in \Sigma_{\mathbf{R}}^{2}$, where $S_{(0,-\infty)} \in \bigcap_{L} \Sigma_{\mathbf{R}}^{L}=\mathscr{S}\left(\mathbf{R}^{2 n}\right)$.

In what follows, we shall investigate that $S_{\kappa} \odot T_{\kappa}$ belongs to $\Sigma_{\mathbf{R}}^{2}$. For the construction of $S_{\kappa} \odot T_{\kappa}$, we have to solve the equation (5.7). Thus, we consider the inverse image of the mapping

$$
\hat{\boldsymbol{\Phi}}\left(\begin{array}{c}
\bar{x} \\
\bar{\xi} \\
\bar{x} \\
\xi
\end{array}\right)=\left(\begin{array}{c}
\bar{x} \\
-\bar{\xi}+\nabla_{\bar{x}} T_{\kappa}(\bar{x}, \xi) \\
-\bar{x}+\nabla_{\bar{\xi}} S_{\kappa}(\bar{x}, \bar{\xi}) \\
\xi
\end{array}\right)
$$

for the domain

$$
\mathbf{R}_{(\bar{x}, \xi)}^{2 n}=\mathbf{R}_{\bar{x}}^{n} \times\{0\} \times\{0\} \times \boldsymbol{R}_{\xi}^{n} .
$$

In preparation for treating (5.17), we will need several notions as follows:

Let $\Sigma_{\mathbf{R}}^{1}\left(\mathbf{R}^{2 n}\right)$ be the space of all mappings

$$
\Psi=\left(\begin{array}{c}
\Psi^{(1)} \\
\vdots \\
\Psi^{(2 n)}
\end{array}\right): \quad \mathbf{R}^{2 n} \rightarrow \mathbf{R}^{2 n}
$$


such that $\Psi^{(i)} \in \Sigma_{\mathbf{R}}^{1}\left(\mathbf{R}^{2 n}\right)$ for any $i, 1 \leq i \leq 2 n . \Sigma_{\mathbf{R}}^{1}\left(\mathbf{R}^{2 n}\right)$ is a Fréchet space under the product topology of $\Sigma_{\mathbf{R}}^{1}\left(\mathbf{R}^{2 n}\right)$. Any element $\Psi \in \Sigma_{\mathbf{R}}^{1}\left(\mathbf{R}^{2 n}\right)$ is written in the shape

$$
\Psi=\Psi_{1}+\Psi_{(0,-L)},
$$

where

$$
\Psi_{1}=\left(\begin{array}{c}
\Psi_{1}^{(1)} \\
\vdots \\
\Psi_{1}^{(2 n)}
\end{array}\right), \quad \Psi_{(0,-L)}=\left(\begin{array}{c}
\Psi_{(0,-L)}^{(1)} \\
\vdots \\
\Psi_{(0,-L)}^{(2 n)}
\end{array}\right)
$$

with

$$
\begin{aligned}
& \Psi_{1}^{(i)}=\Psi_{1}^{(i)}(\omega) \rho \quad \text { (outside some compact subset), } \\
& \left.\Psi_{-l}^{(i)}=\Psi_{-l}^{(i)}(\omega) \rho^{-i} \quad \text { (outside some compact subset, } l=0, \cdots, L-1\right), \\
& \Psi_{-L}^{(i)} \in \mathfrak{B}^{-L} .
\end{aligned}
$$

The identity mapping id: $\mathbf{R}^{2 n} \rightarrow \mathbf{R}^{2 n}$ is obviously an element of $\Sigma_{\mathbf{R}}^{1}\left(\mathbf{R}^{2 n}\right)$.

$\hat{\Phi}^{-1}$ will be computed separately on several conic domains. Here, we give the following notion:

Definition 5.4. Let $\Re$ be positive cones of $\mathbf{R}^{m}-\{0\}$ (i.e. $x \in \Re$ then $t x \in \mathfrak{R}$ for $\forall t \geq 1$ ). For a real valued $C^{\infty}$-function $f$ on $\mathbf{R}^{m}$,

(1) $f \in H^{i}(\mathfrak{R})$ iff $f$ is a homogeneous function of degree $i \in \mathbf{Z}$ on $\boldsymbol{R}$,

(2) $f \in \mathfrak{B}^{-L}(\boldsymbol{\Omega})$ iff $\sup _{w \in \mathcal{R}}\langle w\rangle^{L}\left|\partial^{\alpha} f(w)\right|<\infty$ on $\boldsymbol{\Omega}$,

(3) $f \in \Sigma^{1}(\Omega)$ iff for any $L \in \mathbf{N}$, there exist $f_{1} \in H^{1}(\Omega), \cdots, f_{-L+1} \in H^{-L+1}(\Re)$ such that $f-f_{1}-\cdots f_{-L+1} \in \mathfrak{B}^{-L}(\boldsymbol{\Omega})$,

(4) For a $C^{\infty}$-mapping $\Phi: \mathbf{R}^{m} \rightarrow \mathbf{R}^{m}$, we denote

$$
\Phi=\left(\begin{array}{c}
\Phi^{(1)} \\
\vdots \\
\Phi^{(m)}
\end{array}\right) \in \Sigma^{1}(\boldsymbol{\Omega}) \quad\left(\text { resp. } \in \mathbf{H}^{i}(\boldsymbol{\Omega})\right)
$$

iff $\Phi^{(1)}, \Phi^{(2)}, \cdots, \Phi^{(m)} \in \Sigma^{1}(\Re)\left(\right.$ resp. $\left.\in H^{i}(\Re)\right)$ holds for any $i=1,2, \cdots, m$.

Using these notations, we have the following lemma:

LEMMA 5.5. Let $\Phi: \mathbf{R}^{m} \rightarrow \mathbf{R}^{m}$ be a diffeomorphism. Let $\boldsymbol{R}$ and $\boldsymbol{R}^{\prime}$ be positive cones, such that $\boldsymbol{R}^{\prime} \subset \Phi(\Re) \cap \Phi_{1}(\Re)$, where $\Phi_{1}$ is the part of degree 1 of Definition 5.4 (3). If $\Phi \in \Sigma^{1}(\mathfrak{R})$ and $\Phi_{1}$ is $1-1$, then $\Phi^{-1} \in \Sigma^{1}\left(\Omega^{\prime}\right)$.

Proof. Obviously, we have $\Phi_{1}^{-1} \in \mathbf{H}^{1}\left(\boldsymbol{\Omega}^{\prime}\right)$. Associating to the decomposition in Definition 5.4 (3), we set

$$
\Phi=\Phi_{1}+\Phi_{(0,-L)}, \quad \text { where } \quad \Phi_{(0,-L)}=\Phi_{0}+\cdots+\Phi_{-L+1}+\Phi_{-L},
$$

such that $\Phi_{-l} \in \mathbf{H}^{-l}(\mathfrak{R})(-l \leq 1)$ and $\Phi_{-L} \in \mathfrak{B}^{-L}(\mathfrak{R})$. Then, we have 


$$
\Phi^{-1}=\Phi_{1}^{-1} \circ\left(\Phi-\Phi_{(0,-L)}\right) \circ \Phi^{-1}=\Phi_{1}^{-1} \circ\left(\mathrm{id}-\Phi_{(0,-L)} \circ \Phi^{-1}\right) .
$$

Taking the Taylor expansion of (5.24) at the identity, we have

$$
\begin{aligned}
& \Phi^{-1}=\Phi_{1}^{-1}+\sum_{1 \leq|\alpha| \leq L-1} \frac{1}{\alpha !}\left(-\Phi_{(0,-L)} \circ \Phi^{-1}\right)^{\alpha} \partial^{\alpha} \Phi_{1}^{-1} \\
& +\sum_{|\alpha|=L} \frac{L}{\alpha !}\left(-\Phi_{(0,-L)} \circ \Phi^{-1}\right)^{\alpha} \times \int_{0}^{1}(1-\theta)^{L} \partial^{\alpha} \Phi_{1}^{-1}\left(\mathrm{id}-\theta \Phi_{(0,-L)} \circ \Phi^{-1}\right) d \theta .
\end{aligned}
$$

Here $\Phi_{1} \in \mathbf{H}^{1}\left(\boldsymbol{\Omega}^{\prime}\right)$. The third term of (5.25) is an element of $\mathfrak{B}^{0}\left(\boldsymbol{\Omega}^{\prime}\right)$, since higher order derivatives of the remainder term in (5.25) is bounded on $\Re$. On the other hand, we can easily see the following property:

If $h_{-i} \in H^{-j}\left(\Omega^{\prime}\right)(j=0,1,2, \cdots)$ and

$$
\begin{array}{r}
\Psi^{(i)} \in H^{1}\left(\boldsymbol{\Omega}^{\prime}\right) \oplus H^{0}\left(\boldsymbol{\Omega}^{\prime}\right) \oplus \cdots \oplus H^{-l+1}\left(\boldsymbol{\Omega}^{\prime}\right) \oplus \mathfrak{B}^{-l}\left(\boldsymbol{\Omega}^{\prime}\right) \\
(i=1,2, \cdots, m, l=0,1,2, \cdots),
\end{array}
$$

then

$$
h_{-j} \circ \Psi \in H^{-j}\left(\Omega^{\prime}\right) \oplus H^{-j-1}\left(\Omega^{\prime}\right) \oplus \cdots \oplus H^{-j-l}\left(\Omega^{\prime}\right) \oplus \mathfrak{B}^{-j-l-1}\left(\Omega^{\prime}\right)
$$

Substituting the right hand side of (5.25) to $\Phi^{-1}$ of the right hand side of (5.25), and using the above property, we see that right hand side of (5.25) is contained in $\mathbf{H}^{1}\left(\mathfrak{R}^{\prime}\right) \oplus \mathbf{H}^{0}\left(\boldsymbol{R}^{\prime}\right) \oplus \mathfrak{B}^{-1}\left(\mathfrak{R}^{\prime}\right)$. Hence, we see inductively that right hand side of $(5.25)$ is contained in $\Sigma^{1}\left(\Omega^{\prime}\right)$.

By Lemma 5.3, we have the following result:

LemMA 5.6. Suppose $S, T \in H_{\mathbf{R}}^{2} \oplus H_{\mathbf{R}}^{1}$ are sufficiently close to $x \cdot \xi$. Then, the composed generating function $S_{\kappa} \odot T_{\kappa}$ is an element of $\Sigma_{\mathbf{R}}^{2}$. Furthermore, denoting

$$
\begin{aligned}
S_{\kappa} \odot T_{\kappa} & =\left(S_{\kappa} \odot T_{\kappa}\right)_{2}+\left(S_{\kappa} \odot T_{\kappa}\right)_{1}+\text { lower degree terms } \\
& \in H_{\mathbf{R}}^{2} \oplus H_{\mathbf{R}}^{1} \oplus \Sigma_{\mathbf{R}}^{0},
\end{aligned}
$$

we have

(1) $\left(S_{\kappa} \odot T_{\kappa}\right)_{2}$ depends only on $S_{2}, T_{2}$.

(2) $\left(S_{\kappa} \odot T_{\kappa}\right)_{1}$ depends only on $S_{2}, T_{2}, S_{1}, T_{1}$.

Proof. For short, we write $S, T$ instead of $S_{\kappa}, T_{\kappa}$. As we have mentioned in (5.17), we have to treat the inverse of $\hat{\Phi}$. Consider the following positive cones:

$$
\begin{gathered}
\Re=\{(\bar{x}, \bar{\xi}, \bar{x}, \xi) ;\|\bar{x}, \xi\| \geq K, 1-\varepsilon \leq\|\bar{x}, \bar{\xi}\| /\|\bar{x}, \xi\| \leq 1+\varepsilon\}, \\
\mathfrak{R}^{\prime}=\left\{(\bar{x}, 0,0, \xi) ;\|\bar{x}, \xi\| \geq K^{\prime}\right\} .
\end{gathered}
$$

Since the Jacobian matrix of $\hat{\Phi}$ : 


$$
\begin{aligned}
& D_{(\bar{x}, \bar{\xi}, \bar{x}, \xi)} \hat{\Phi}=\left(\begin{array}{cccc}
E_{n} & 0 & 0 & 0 \\
0 & -E_{n} & \partial_{\bar{x}} \partial_{\bar{x}} T & \partial_{\bar{x}} \partial_{\xi} T \\
\partial_{\bar{x}} \partial_{\xi} S & \partial_{\xi} \partial_{\xi} S & -E_{n} & 0 \\
0 & 0 & 0 & E_{n}
\end{array}\right) \\
& \text { are close to }\left(\begin{array}{cccc}
E_{n} & 0 & 0 & 0 \\
0 & -E_{n} & 0 & E_{n} \\
E_{n} & 0 & -E_{n} & 0 \\
0 & 0 & 0 & E_{n}
\end{array}\right) \text { uniformly on } \mathbf{R}^{4 n},
\end{aligned}
$$

and the same thing holds for the component $\hat{\Phi}_{1}$ of homogeneous degree 1 , we see that on $\boldsymbol{\Omega}^{\prime}$

$$
\|\overline{\bar{x}}, \bar{\xi}(\overline{\bar{x}}, \xi)\| \sim\|\bar{x}(\overline{\bar{x}}, \xi), \bar{\xi}(\overline{\bar{x}}, \xi)\| \sim\|\bar{x}(\overline{\bar{x}}, \xi), \xi\| \sim\|\overline{\bar{x}}, \xi\|,
$$

where $a \sim b$ implies $|1-b / a|<\varepsilon$. Thus, we see that $\boldsymbol{\Re}^{\prime} \subset \hat{\Phi}(\boldsymbol{\Omega}) \cap \hat{\Phi}_{1}(\Re)$. Applying Lemma 5.5 to $\hat{\Phi}$, we have $\hat{\Phi}^{-1} \in \Sigma^{1}\left(\boldsymbol{\Omega}^{\prime}\right)$.

We now compute $S \odot T$. Denote the mapping $\hat{\Phi}$ by

$$
\hat{\boldsymbol{\Phi}}\left(\begin{array}{c}
\overline{\bar{x}} \\
\bar{\xi} \\
\bar{x} \\
\xi
\end{array}\right)=\left(\begin{array}{c}
\bar{y} \\
\bar{\eta} \\
\bar{y} \\
\eta
\end{array}\right)=\left(\begin{array}{c}
\mathfrak{C}_{\bar{y}}(\hat{\Phi}) \\
\mathfrak{C}_{\bar{\eta}}(\hat{\Phi}) \\
\mathfrak{C}_{\bar{y}}(\hat{\Phi}) \\
\mathfrak{C}_{y}(\hat{\Phi})
\end{array}\right) .
$$

Similarly, we set

$$
\hat{\Phi}^{-1}\left(\begin{array}{c}
\bar{y} \\
\bar{\eta} \\
\bar{y} \\
\eta
\end{array}\right)=\left(\begin{array}{l}
\mathfrak{C}_{\bar{x}}\left(\hat{\Phi}^{-1}\right) \\
\mathfrak{C}_{\bar{\xi}}\left(\hat{\Phi}^{-1}\right) \\
\mathfrak{C}_{\bar{x}}\left(\hat{\Phi}^{-1}\right) \\
\mathfrak{C}_{x}\left(\hat{\Phi}^{-1}\right)
\end{array}\right)
$$

If the variables are restricted in the space $\mathbf{R}_{(\overline{\bar{x}}, \xi)}^{2 n}$, then we denote also

$$
\widetilde{\mathfrak{C}}_{\bar{\xi}}\left(\hat{\Phi}^{-1}\right)=\left.\mathfrak{C}_{\bar{\xi}}\left(\hat{\Phi}^{-1}\right)\right|_{\mathbf{R}_{(\bar{x}, \xi)}^{2 n},}, \quad \widetilde{\mathfrak{C}}_{\bar{x}}\left(\hat{\Phi}^{-1}\right)=\left.\mathfrak{C}_{\bar{x}}\left(\hat{\Phi}^{-1}\right)\right|_{\mathbf{R}_{(\bar{x} \xi)}^{2 n}}, \quad \text { etc. }
$$

By using these notations, we can rewrite $S \odot T$ in (5.11) as follows:

$$
S \odot T(\bar{x}, \xi)=S\left(\bar{x}, \widetilde{\mathbb{C}}_{\bar{\xi}}\left(\hat{\Phi}^{-1}\right)\right)-\left\langle\widetilde{\mathbb{C}}_{\bar{\xi}}\left(\hat{\Phi}^{-1}\right) \mid \widetilde{\mathbb{C}}_{\bar{x}}\left(\hat{\Phi}^{-1}\right)\right\rangle+T\left(\widetilde{\mathbb{C}}_{\bar{x}}\left(\hat{\Phi}^{-1}\right), \xi\right) .
$$

Restricting $\hat{\Phi}^{-1}$ to $\mathbf{R}_{(\bar{x}, \xi)}^{2 n}$, we see that the mapping

$$
F(S, T)=\left(\widetilde{\mathbb{C}}_{\bar{\xi}}\left(\hat{\Phi}^{-1}\right), \widetilde{\mathfrak{C}}_{\bar{x}}\left(\hat{\Phi}^{-1}\right)\right): \mathbf{R}_{(\bar{x}, \xi)}^{2 n} \rightarrow \mathbf{R}_{(\bar{x}, \bar{\xi})}^{2 n}
$$

is contained in $\Sigma_{\mathbf{R}}^{1}\left(\mathbf{R}^{2 n}\right)$.

Recall that $\hat{\Phi}$ depends on $S, T$. Note that there exists a neighborhood $U_{x, \xi}$ of $x \cdot \xi$ in $H_{\mathbf{R}}^{2} \oplus H_{\mathbf{R}}^{1}$ such that $\hat{\Phi}$ satisfies the condition of Lemma 5.1 for any $S, T \in U_{x \cdot \xi}$ 
Therefore, $F$ given in (5.31) is regarded as a mapping of $U_{x \cdot \xi} \times U_{x \cdot \xi}$ into $\Sigma_{\mathbf{R}}^{1}\left(\mathbf{R}^{2 n}\right)$. In $\S 7$, we shall show the smoothness of the mapping $F$ (cf. Lemma 7.6).

We compute the decomposition of $F$. For simplicity, we put

$$
(\bar{\xi}, \bar{x})=\left(\widetilde{\mathbb{C}}_{\bar{\xi}}\left(\hat{\Phi}^{-1}\right)(\bar{x}, \xi), \widetilde{\boldsymbol{C}}_{\bar{x}}\left(\hat{\Phi}^{-1}\right)(\bar{x}, \xi)\right),
$$

and set

$$
\begin{array}{ll}
\bar{\xi}=\bar{\xi}_{1}+\bar{\xi}_{0}+\cdots, & \bar{\xi}_{j}^{(i)} \in H_{\mathbf{R}}^{j}, \\
\bar{x}=\bar{x}_{1}+\bar{x}_{0}+\cdots, & \bar{x}_{j}^{(i)} \in H_{\mathbf{R}}^{i} .
\end{array}
$$

It is easily seen that $\bar{\xi}_{1}(\bar{x}, \xi)$ and $\bar{x}_{1}(\bar{x}, \xi)$ depend only on $S_{2}, T_{2}$ and $\bar{\xi}_{0}(\bar{x}, \xi)$ and $\bar{x}_{0}(\bar{x}, \xi)$ depend only on $S_{2}, T_{2}, S_{1}, T_{1}$. Substituting (5.32) to (5.30), we have

$$
\begin{aligned}
(S \odot T)_{2}= & S_{2}\left(\bar{x}, \bar{\xi}_{1}(\bar{x}, \xi)\right)-\left\langle\bar{\xi}_{1}(\bar{x}, \xi) \mid \bar{x}_{1}(\bar{x}, \xi)\right\rangle+T_{2}\left(\bar{x}_{1}(\bar{x}, \xi), \xi\right) \\
(S \odot T)_{1}= & \left(\nabla_{\bar{\xi}} S_{2}\right)\left(\bar{x}, \bar{\xi}_{1}(\bar{x}, \xi)\right) \cdot \bar{\xi}_{0}(\bar{x}, \xi)+S_{1}\left(\bar{x}, \bar{\xi}_{1}(\bar{x}, \xi)\right) \\
& -\left\langle\bar{\xi}_{1}(\bar{x}, \xi) \mid \bar{x}_{0}(\bar{x}, \xi)\right\rangle-\left\langle\bar{\xi}_{0}(\bar{x}, \xi) \mid \bar{x}_{1}(\bar{x}, \xi)\right\rangle \\
& +T_{1}\left(x_{1}(\bar{x}, \xi), \xi\right)+\left(\nabla_{\bar{x}} T_{2}\right)\left(\bar{x}_{1}(\bar{x}, \xi), \xi\right) \cdot \bar{x}_{0}(\bar{x}, \xi) .
\end{aligned}
$$

Hence, we obtain the results.

Next, we shall investigate the generating function of inverse mapping $\varphi_{S}^{-1}$. Let $\chi_{\varphi_{S}^{-1}}$ be the cross mapping corresponding to $\varphi_{S}^{-1}$

$$
\begin{aligned}
\varphi_{S}^{-1}: & \begin{array}{c}
\mathbf{R}_{(x, \xi)}^{2 n} \\
\Psi
\end{array} \\
\left(\begin{array}{c}
\bar{x} \\
\nabla_{\bar{x}} S(\bar{x}, \xi)
\end{array}\right) & \longmapsto \begin{array}{c}
\mathbf{R}_{(\bar{x}, \bar{\xi})}^{2 n} \\
\Psi
\end{array}
\end{aligned}
$$

Applying Lemma 5.2 to $\chi_{\varphi_{S}^{-1}}$, we get the generating function of the cross mapping $\chi_{\varphi_{S}^{-1}}$. Next, we set

$$
\hat{S}=\hat{S}_{2} \oplus \hat{S}_{1}=\text { the } H_{\mathbf{R}}^{2} \oplus H_{\mathbf{R}}^{1} \text {-part of } \sigma_{\varphi_{S}^{-1}} .
$$

If we make the canonical transformation corresponding to $\hat{S}$, then by Lemma 5.6, we see the following lemma:

LEMMA 5.7. Under the above notations, the homogeneous term of degree 1 of $S \odot \hat{S}$ vanishes, i.e.

$$
\begin{aligned}
S \odot \hat{S}=x \cdot \xi+0 & +\{\text { homogeneous term of degree } 0\} \\
+ & \{\text { homogeneous term of degree }-1\}+\cdots .
\end{aligned}
$$

In the last part of this section, we investigate relations among contact transformations, canonical transformations and generating functions.

In what follows, we shall construct a mapping $p_{1}$ of a star-shaped neighborhood $\hat{U}$ of the identity of $\mathscr{D}_{\omega}\left(S^{2 n-1}\right)$ into the group $\mathscr{D}_{\Omega}\left(\mathbf{R}^{2 n}\right)$ of all canonical transformations 
on $\mathbf{R}^{2 n}$. For that purpose, we take $\hat{U}$ very small so that Lemma 5.1 can be applied to the cross mapping to obtain canonical transformations. Next, let $\tilde{\varphi}_{t}$ be a curve in $\hat{U}$ such that $\tilde{\varphi}_{0}=\mathrm{id}, \tilde{\varphi}_{1}=\tilde{\varphi}$. Since $\tilde{\varphi}_{t} \in \mathscr{D}_{\omega}\left(S^{2 n-1}\right)_{0}$, there exists $h_{t} \in C^{\infty}\left(S^{2 n-1}\right)$ such that $\tilde{\varphi}_{t}^{*} \theta=h_{t} \theta$ where $\theta$ is the standard contact 1 -form on $S^{2 n-1}$, and $\tilde{\varphi}_{t}^{*} \theta$ is a pull back of $\theta$ by $\tilde{\varphi}_{t}$.

Under the above notations, we define $\varphi$ corresponding to $\tilde{\varphi}$ as follows:

$$
\begin{aligned}
& \mathbf{R}^{2 n}=\mathbf{R}_{+} \times S^{2 n-1} \stackrel{\varphi}{\longrightarrow} \quad \mathbf{R}_{+} \times S^{2 n-1} \\
& \boldsymbol{\omega} \\
& (\rho, \omega) \longmapsto\left(f(\rho, \omega), \tilde{\varphi}_{\kappa(2 \rho)}(\omega)\right),
\end{aligned}
$$

where

$$
f(\rho, \omega)=\rho / h_{\kappa(2 \rho)}(\omega)
$$

and $\kappa$ is defined in (3.2).

Then $p_{1}$ is defined by $p_{1}(\tilde{\varphi})=\varphi . \quad p_{1}$ is a mapping of $\hat{U}$ into $\mathscr{D}_{\Omega}\left(\mathbf{R}^{2 n}\right)$, and $p_{1}(\tilde{\varphi})$ is positively homogeneous degree 1 outside some compact subset on $\mathbf{R}^{2 n}$.

LEMMA 5.8 .

$$
\begin{aligned}
& \text { If } \rho \leq 1 / 4, \text { then } \tilde{\varphi}_{\kappa(2 \rho)}(\omega)=\mathrm{id}(\omega), h_{\kappa(2 \rho)}(\omega)=1 . \\
& \text { If } \rho \geq 1-1 / 4, \text { then } \tilde{\varphi}_{\kappa(2 \rho)}(\omega)=\tilde{\varphi}(\omega), h_{\kappa(2 \rho)}(\omega)=h_{1}(\omega) \text {. }
\end{aligned}
$$

Next, we shall define a mapping $p_{2}$ which gives the correspondence between elements of $\operatorname{Im} p_{1}$ and generating functions.

Applying Lemma 5.1, we solve $\bar{x}=\varphi_{\bar{x}}(x, \xi)$ with respect to $x$ and set $x=x(\bar{x}, \xi)$, where we write

$$
\varphi=\left(\varphi_{\bar{x}}, \varphi_{\bar{\xi}}\right): \mathbf{R}_{(x, \xi)}^{2 n} \rightarrow \mathbf{R}_{(\bar{x}, \bar{\xi})}^{2 n} .
$$

By substituting this to $\bar{\xi}=\varphi_{\xi}(x, \xi)$ we obtain the cross mapping $(x, \bar{\xi})=\chi(\bar{x}, \xi)$ which corresponds to the canonical transformation $\varphi(x, \xi)=(\bar{x}, \bar{\xi})$. By Lemma 5.2, we obtain the generating function $S_{\varphi}$ of $\varphi$ by

$$
S_{\varphi}(\bar{x}, \xi)=\int_{0}^{1}\{\bar{\xi}(t \bar{x}, t \xi) \cdot \bar{x}+x(t \bar{x}, t \xi) \cdot \xi\} d t \in \kappa\left\{H_{\mathbf{R}}^{2}+H_{\mathbf{R}}^{0}\right\}+\mathscr{S} .
$$

Using these notations, we define a mapping $p_{2}$ by

$$
p_{2}: \operatorname{Im} p_{1} \ni \varphi \mapsto S_{\varphi} \in \kappa\left\{H_{\mathbf{R}}^{2}+H_{\mathbf{R}}^{0}\right\}+\mathscr{S} .
$$

By the construction of (5.40), (5.38), we see that the mapping

$$
\hat{U} \ni \hat{\varphi} \mapsto S_{\varphi} \in \kappa\left\{H_{\mathbf{R}}^{2}+H_{\mathbf{R}}^{0}\right\}+\mathscr{S}
$$

is $C^{\infty}$. 
Conversely, $S_{2}$ which is close to $x \cdot \xi$ in $H_{\mathbf{R}}^{2}$ corresponds to contact transformation $\tilde{\varphi}_{S_{2}}$. Though we use $\kappa\left\{H_{\mathbf{R}}^{2}+H_{\mathbf{R}}^{0}\right\}+\mathscr{S}$ in the process of making canonical transformations, there is a bijective correspondence between the neighborhood of $x \cdot \xi \in H_{\mathbf{R}}^{2}$ and the neighborhood of the identity in $\mathscr{D}_{\omega}\left(S^{2 n-1}\right)_{0}$.

\section{Compositions of inhomogeneous Fourier integral operators.}

In this section, we shall consider the composition of inhomogeneous Fourier integral operators. We want to rewrite the composition

$$
F\left(a, S_{1}, \tilde{\varphi}_{S_{2}}\right) \circ F\left(b, T_{1}, \tilde{\varphi}_{T_{2}}\right)
$$

in the shape of (1.4) by reducing the number of variables.

For that purpose, we employ the result of Asada-Fujiwara [AF] which will be mentioned below:

Consider the oscillatory integral

$$
A(v) f(x)=O s-\iint_{\mathbf{R}^{m} \times \mathbf{R}^{n}} s(x, \theta, y) e^{(\sqrt{-1} v \psi(x, \theta, y)} f(y) d y d \theta
$$

with the following conditions:

(A-1) $\psi(x, \theta, y)$ is a real valued $C^{\infty}$-function of $(x, \theta, y) \in \mathbf{R}^{n} \times \mathbf{R}^{m} \times \mathbf{R}^{n}$.

(A-2) There exists a positive constant $\delta_{0}$ such that $|\operatorname{det} D(\psi)(x, \theta, y)| \geq \delta_{0}$, where $D(\psi)(x, \theta, y)$ is the $(m+n)$ square matrix

$$
D(\psi)(x, \theta, y)=\left(\begin{array}{ll}
\partial^{2} \psi(x, \theta, y) / \partial x \partial y & \partial^{2} \psi(x, \theta, y) / \partial x \partial \theta \\
\partial^{2} \psi(x, \theta, y) / \partial \theta \partial y & \partial^{2} \psi(x, \theta, y) / \partial \theta \partial \theta
\end{array}\right) .
$$

(A-3) Every component of the matrix $D(\psi)(x, \theta, y)$ belongs to the space $\mathscr{B}\left(\mathbf{R}^{n} \times \mathbf{R}^{m} \times \mathbf{R}^{n}\right)$ of Schwartz, the space of all $f$ such that for any multi-indices $\alpha, \beta, \gamma$, there exists a positive constant $C_{\alpha, \beta, \gamma}$ such that

$$
\left|\partial_{x}^{\alpha} \partial_{y}^{\beta} \partial_{\theta}^{\gamma} f(x, \theta, y)\right| \leq C_{\alpha, \beta, \gamma} .
$$

(A-4) The amplitude function $s$ belongs to the space $\mathscr{B}\left(\mathbf{R}^{n} \times \mathbf{R}^{m} \times \mathbf{R}^{n}\right)$.

Suppose that the variable $\theta \in \mathbf{R}^{m}$ splits into two class of variables $\theta^{\prime} \in \mathbf{R}^{m_{1}}, \theta^{\prime \prime} \in \mathbf{R}^{m-m_{1}}$ (i.e. $\theta=\left(\theta^{\prime}, \theta^{\prime \prime}\right)$ ), and $\psi$ satisfies the following:

(A-5) There exists a positive constant $\delta^{\prime}$ such that

$$
\left|\operatorname{det} \frac{\partial^{2}}{\partial \theta^{\prime \prime} \partial \theta^{\prime \prime}} \varphi\left(x, \theta^{\prime}, \theta^{\prime \prime}, y\right)\right| \geq \delta^{\prime}
$$

holds at every $\left(x, \theta^{\prime}, \theta^{\prime \prime}, y\right) \in \mathbf{R}^{n} \times \mathbf{R}^{m} \times \mathbf{R}^{n}$.

By Lemma 5.1, it is seen that the equation $\left(\partial / \partial \theta^{\prime \prime}\right) \psi\left(x, \theta^{\prime}, \theta^{\prime \prime}, y\right)=0$ has the unique solution $\theta^{\prime \prime}=\theta^{\prime \prime}\left(x, \theta^{\prime}, y\right)$, and the first derivatives of $\theta^{\prime \prime}\left(x, \theta^{\prime}, y\right)$ belong to the class 
$\mathscr{B}\left(\mathbf{R}^{n} \times \mathbf{R}^{m_{1}} \times \mathbf{R}^{n}\right)$. Using this, we set

$$
\begin{aligned}
\psi_{0}\left(x, \theta^{\prime}, y\right) & =\psi\left(x, \theta^{\prime}, \theta^{\prime \prime}\left(x, \theta^{\prime}, y\right), y\right), \\
s^{\prime}\left(x, \theta^{\prime}, y\right) & =s\left(x, \theta^{\prime}, \theta^{\prime \prime}\left(x, \theta^{\prime}, y\right), y\right) .
\end{aligned}
$$

Furthermore, we see that the new phase function $\psi_{0}$ satisfies $(\mathrm{A}-1 \sim 3)$, and $s^{\prime}$ satisfies (A-4).

THEOREM 6.1 (Asymptotic reduction theorem [AF]). With the notations and assumptions as above the integral transformation (6.2) is rewritten as

$$
A(v) f(x)=O s-\iint_{\mathbf{R}^{m_{1} \times \mathbf{R}^{n}}} s^{\prime}\left(v, x, \theta^{\prime}, y\right) e^{\sqrt{-1} v \psi_{0}\left(x, \theta^{\prime}, y\right)} f(y) d y d \theta^{\prime} .
$$

For the detailed computation of $s^{\prime}$, we put

$$
\begin{gathered}
H\left(x, \theta^{\prime}, y\right)=\left.\frac{\partial^{2}}{\partial \theta^{\prime \prime} \partial \theta^{\prime \prime}} \psi\left(x, \theta^{\prime}, \theta^{\prime \prime}, y\right)\right|_{\theta^{\prime \prime}=\theta^{\prime \prime}\left(x, \theta^{\prime}, y\right)} \\
h(x, \theta, y)= \\
\psi(x, \theta, y)-\psi_{0}\left(x, \theta^{\prime}, y\right) \\
-\frac{1}{2}\left\langle H\left(x, \theta^{\prime}, y\right)\left(\theta^{\prime \prime}-\theta^{\prime \prime}\left(x, \theta^{\prime}, y\right)\right),\left(\theta^{\prime \prime}-\theta^{\prime \prime}\left(x, \theta^{\prime}, y\right)\right)\right\rangle .
\end{gathered}
$$

To state the second assertion of $[\mathrm{AF}]$, we use the following notations:

$$
\begin{gathered}
e_{N}^{\Omega}=\sum_{k=0}^{N} \frac{1}{k !} \Omega^{k}, \\
\mathfrak{I}_{m-m_{1}}\left(x, \theta^{\prime}, y\right)=\left|\operatorname{det} H\left(x, \theta^{\prime}, y\right)\right|^{-1 / 2} e^{(\pi \sqrt{-1} / 4)\left(m-m_{1}-2 \operatorname{Ind} H\left(x, \theta^{\prime}, y\right)\right)} .
\end{gathered}
$$

THEOREM 6.2 (Asymptotic expansion formula $[\mathrm{AF}]$ ). For any integer $N \geq 1$,

$$
\begin{aligned}
s^{\prime}(v, x, & \left.\theta^{\prime}, y\right) \\
= & \left.(2 \pi / v)^{\left(m-m_{1}\right) / 2} \mathfrak{J}_{m-m_{1}}\left(x, \theta^{\prime}, y\right)\left\{e_{N}^{\Omega}\left(s(x, \theta, y) e^{\sqrt{-1} v h(x, \theta, y)}\right)\right\}\right|_{\theta^{\prime \prime}=\theta^{\prime \prime}\left(x, \theta^{\prime}, y\right)} \\
& +r_{N+1}\left(v, x, \theta^{\prime}, y\right),
\end{aligned}
$$

where $\Omega$ stands for the operator

$$
-\frac{\sqrt{-1}}{2 v} \sum_{i, j}\left(\left.\frac{\partial^{2}}{\partial \theta^{\prime \prime} \partial \theta^{\prime \prime}} \psi\left(x, \theta^{\prime}, \theta^{\prime \prime}, y\right)\right|_{\theta^{\prime \prime}=\theta^{\prime \prime}\left(x, \theta^{\prime}, y\right)}\right)_{i, j}^{-1} \partial_{\theta_{i}^{\prime \prime}} \partial_{\theta_{j}^{\prime \prime}},
$$

and $r_{N+1}$ satisfies for any multi-indices $\alpha, \beta$ and $\gamma$, there exists a positive constant $C_{\alpha, \beta, \gamma}$ such that

$$
\left|\partial_{x}^{\alpha} \partial_{y}^{\beta} \partial_{\theta^{\gamma}}^{\gamma} r_{N+1}\left(x, \theta^{\prime}, y\right)\right| \leq C_{\alpha, \beta, \gamma} \nu^{-N-1}
$$

To apply Theorems 6.1 and 6.2 to our situation, $v, x, \theta, y, \theta^{\prime \prime}, \theta^{\prime}$ are replaced by $\hbar^{-1}, \bar{x},(\bar{\xi}, \bar{x}, \xi), x,(\bar{\xi}, \bar{x}), \xi$, and we set as follows: 


$$
\begin{gathered}
s(x, \theta, y)=a(\bar{x}, \bar{\xi}) b(\bar{x}, \xi), \\
S(\bar{x}, \bar{\xi})=\kappa\left\{S_{2}(\bar{x}, \bar{\xi})+S_{1}(\bar{x}, \bar{\xi})\right\}+(1-\kappa) \bar{x} \cdot \bar{\xi}, \\
T(\bar{x}, \xi)=\kappa\left\{T_{2}(\bar{x}, \xi)+T_{1}(\bar{x}, \xi)\right\}+(1-\kappa) \bar{x} \cdot \xi, \\
\psi=S(\bar{x}, \bar{\xi})-\bar{\xi} \cdot \bar{x}+T(\bar{x}, \xi)-\xi \cdot x .
\end{gathered}
$$

Then, we see that the assumptions (A-1 5) of Theorems 6.1 and 6.2 are satisfied. To check (A-5), we use the assumption that both $S$ and $T$ of (6.11) are close to $\bar{x} \cdot \bar{\xi}$ and $\bar{x} \cdot \xi$ in $H_{\mathbf{R}}^{2} \oplus H_{\mathrm{R}}^{1}$ respectively. Hence, we can apply Theorems 6.1 and 6.2 to the following integral:

$$
\begin{aligned}
& F\left(a, S_{1}, \tilde{\varphi}_{S_{2}}\right) \circ F\left(b, T_{1}, \tilde{\varphi}_{T_{2}}\right) u(\bar{x}) \\
& \quad=O s-\iiint \int a(\bar{x}, \bar{\xi}) b(\bar{x}, \xi) e^{(\sqrt{-1} / \hbar)\{S(\bar{x}, \bar{\xi})-\bar{\xi} \cdot \bar{x}+T(\bar{x}, \xi)-\xi \cdot x\}} u(x) \bar{d} x \bar{d} \xi \bar{d} \bar{x} \bar{d} \bar{\xi}
\end{aligned}
$$

At first, take the solution $(\bar{x}(\bar{x}, \xi), \bar{\xi}(\bar{x}, \xi))$ of

$$
\left\{\begin{array}{l}
\bar{x}-\nabla_{\bar{\xi}} S(\bar{x}, \bar{\xi})=0 \\
\bar{\xi}-\nabla_{\bar{x}} T(\bar{x}, \xi)=0,
\end{array}\right.
$$

and remark that $\psi_{0}$ in (6.3) is given by

$$
\psi_{0}(\bar{x}, \xi, x)=\psi(\bar{x}, \bar{\xi}(\bar{x}, \xi), \bar{x}(\bar{x}, \xi), \xi, x)=S \odot T(\bar{x}, \xi)-\xi \cdot x
$$

Substituting (6.8) and (6.14) to (6.4), we see that (6.12) equals

$$
\begin{array}{r}
O s-\iint\left[\left.\mathfrak{I}_{2 n}(\bar{x}, \xi)\left\{e_{N}^{\Omega}\left(a(\bar{x}, \bar{\xi}) b(\bar{x}, \xi) e^{(\sqrt{-1} / \hbar) h(\bar{x}, \bar{\xi}, \bar{x}, \xi)}\right)\right\}\right|_{\substack{\bar{\xi}=\bar{\xi}(\bar{x}, \xi) \\
\bar{x}=\bar{x}(\bar{x}, \xi)}}\right. \\
\left.+p_{N+1}(\bar{x}, \xi ; \hbar)\right] e^{(\sqrt{-1 / \hbar n)(S \odot T(\bar{x}, \xi)-\xi \cdot x\}} u(x) d x d \xi},
\end{array}
$$

where $\Omega$ stands for (6.9) and $p_{N+1}$ in the last line is a function satisfying

$$
\left|\partial_{\bar{x}}^{\alpha} \partial_{\xi}^{\beta} p_{N+1}(\bar{x}, \xi ; \hbar)\right| \leq C_{\alpha, \beta}\langle x ; \xi\rangle^{-2 N-2} \text {. }
$$

We remark also that $\mathfrak{I}_{2 n}(\bar{x}, \xi)$ in the first line should be written $\mathfrak{I}_{2 n}(\bar{x}, \xi, x)$ in the context of (6.7). Since $\mathfrak{I}_{2 n}$ does not involve $x$-variable, we omit this.

The following lemma shows the uniqueness of our expression of Fourier integral operators:

LEMMA 6.3. If $a, b \in \Sigma_{\mathbf{C}}^{0}, S_{1}, T_{1} \in H_{\mathrm{R}}^{1}, S_{2}, T_{2} \in H_{\mathrm{R}}^{2}$, we assume that $a, b$ are close to $1, S_{2}, T_{2}$ are close to $x \cdot \xi$. If $F\left(a, S_{1}, \tilde{\varphi}_{S_{2}}\right)=F\left(b, T_{1}, \tilde{\varphi}_{T_{2}}\right)$ then $T_{2}=S_{2}, S_{1}=T_{1}$, $a(\bar{x}, \xi)=b(\bar{x}, \xi)$ hold. 
Proof. Suppose $F\left(a, S_{1}, \tilde{\varphi}_{S_{2}}\right)=F\left(b, T_{1}, \tilde{\varphi}_{T_{2}}\right)$. Then for any $\hat{u} \in \mathscr{S}\left(\mathbf{R}^{2 n}\right)$, we have

$$
\int a(\bar{x}, \xi) e^{(\sqrt{-1} / \hbar)\left\{S_{2}+S_{1}\right\}(\bar{x}, \xi)} \hat{u}(\xi) \bar{d} \xi=\int b(\bar{x}, \xi) e^{(\sqrt{-1} / \hbar)\left\{T_{2}+T_{1}\right\}(\bar{x}, \xi)} \hat{u}(\xi) d \xi,
$$

hence

$$
a(\bar{x}, \xi) e^{(\sqrt{-1} / \hbar)\left\{S_{2}+S_{1}\right\}(\bar{x}, \xi)}=b(\bar{x}, \xi) e^{(\sqrt{-1} / \hbar)\left\{T_{2}+T_{1}\right\}(\bar{x}, \xi)},
$$

as integrand are continuous functions. Thus, we have

$$
a(\bar{x}, \xi) / b(\bar{x}, \xi)=e^{(\sqrt{-1} / \hbar)\left\{T_{2}+T_{1}-S_{2}-S_{1}\right\}} .
$$

Left hand side is in $\Sigma_{\mathbf{C}}^{0}$. Comparing the behavior of both sides of (6.17) as $\rho \rightarrow \infty$ with taking (6.11) into account, we have $T_{2}=S_{2}, S_{1}=T_{1}$, and then $a(\bar{x}, \xi)=b(\bar{x}, \xi)$.

We obtain the composition rule as follows.

LEMMA 6.4 .

$$
F\left(a, S_{1}, \hat{\varphi}_{S_{2}}\right) \circ F\left(b, T_{1}, \tilde{\varphi}_{T_{2}}\right)=F\left(c\left(a, S_{1}, \tilde{\varphi}_{S_{2}}, b, T_{1}, \tilde{\varphi}_{T_{2}}\right),(S \odot T)_{1}, \tilde{\varphi}_{(S \odot T)_{2}}\right),
$$

where

$$
\begin{aligned}
c\left(a, S_{1},\right. & \left.\tilde{\varphi}_{S_{2}}, b, T_{1}, \tilde{\varphi}_{T_{2}}\right)(\bar{x}, \xi) \\
= & {\left[\left.I_{2 n}(\bar{x}, \xi)\left\{e_{N}^{\Omega}\left(a(\bar{x}, \bar{\xi}) b(\bar{x}, \xi) e^{(\sqrt{-1} / \hbar) h(\bar{x}, \bar{\xi}, \bar{x}, \xi)}\right)\right\}\right|_{\substack{\bar{\xi}=\bar{\xi}(\overline{\bar{x}}, \xi) \\
\bar{x}=\bar{x}(\bar{x}, \xi)}}+p_{N+1}(\bar{x}, \xi ; \hbar)\right] } \\
& \times e^{(i / \hbar)\left(S \odot T(\bar{x}, \xi)-\kappa\left((S \odot T)_{2} \oplus(S \odot T)_{1}\right)(\bar{x}, \xi)\right\}} \in \sum_{\mathbf{C}}^{0},
\end{aligned}
$$

and $\Omega$ stands for the operator (6.9). A generating function $S \odot T$ satisfies

$$
S \odot T-\kappa\left\{(S \odot T)_{2}+(S \odot T)_{1}\right\} \in \Sigma_{\mathbf{R}}^{0} .
$$

We now compute the inverse of inhomogeneous Fourier integral operators.

Given an inhomogeneous Fourier integral operator $F\left(a, S_{1}, \tilde{\varphi}_{S_{2}}\right)$ such that $S=$ $S_{2}+S_{1}$ is an element of $U_{x \cdot \xi}$ in (5.31), there exists $\hat{S} \in H_{\mathbf{R}}^{2} \oplus H_{\mathbf{R}}^{1}$ satisfying (5.36) by Lemma 5.7. Then, by Lemma 6.4, $F\left(1, \hat{S}_{1}, \tilde{\varphi}_{S_{2}}\right)$ satisfies

$$
F\left(1, \hat{S}^{(1)}, \tilde{\varphi}_{S_{2}}\right) \circ F\left(a, S_{1}, \hat{\varphi}_{S_{2}}\right)=F\left(c, 0, \hat{\varphi}_{x \cdot \xi}\right) \in G F^{0} \text {. }
$$

By Proposition A and Lemma 4.3, we see that if $S$ is sufficiently close to $x \cdot \xi$ then $F\left(c, 0, \hat{\varphi}_{x \cdot \xi}\right)$ is an invertible pseudo-differential operator contained in $G \Sigma^{0}$. We shall denote $F\left(c, 0, \hat{\varphi}_{x \cdot \xi}\right)^{-1}$ by $P_{S}$. Hence, we see the following:

LEMMA 6.5. Under the above notations,

$$
F\left(a, S_{1}, \tilde{\varphi}_{S_{2}}\right)^{-1}=P_{S^{\circ}} F\left(1, \hat{S}_{1}, \tilde{\varphi}_{S_{2}}\right) \in G F^{0} .
$$

Combining Lemma 6.4 with Lemma 6.5, we have the following lemma:

LEMMA 6.6. $G \Sigma^{0}$ is a normal subgroup of $G F^{0}$. 
Therefore we can define the quotient group $G F^{0} / G \Sigma^{0}$, which is called the phase group.

\section{Proof of Proposition B.}

Before showing Proposition B, we need several preparatory lemmas.

We use notations defined in $\S 5$. The following lemma is related to the smoothness of compositions.

LEMMA 7.1. There exists a neighborhood $U_{\mathrm{id}}$ of identity of $\Sigma_{\mathbf{R}}^{1}\left(\mathbf{R}^{2 n}\right)$ such that the following mapping is smooth:

$$
c: \Sigma_{\mathbf{K}}^{-m} \times U_{\text {id }} \ni(a, \Phi) \mapsto a \circ \Phi \in \Sigma_{\mathbf{K}}^{-m},
$$

where $m=-1,0,1,2, \cdots$.

Proof. $\quad c(a, \Phi)=a \circ \Phi$ is linear in $a$, hence smooth in $a \in \Sigma_{\mathbf{K}}^{-m}$. So, it is enough to see by Proposition 2.2 (III), that $c(a, \Phi)$ is smooth in $\Phi$.

Now, set

$$
\delta \Phi=\left(\begin{array}{c}
\delta \Phi^{(1)} \\
\vdots \\
\delta \Phi^{(2 n)}
\end{array}\right)=\delta \Phi_{1}+\delta \Phi_{(0,-L)} \in \Sigma_{\mathbf{R}}^{1}
$$

Using the Taylor's theorem, we obtain

(7.3) $c(a, \Phi+\delta \Phi)(x, \xi)=(a \circ \Phi)(x, \xi)+\sum_{1 \leq|\alpha| \leq N} \frac{1}{r !}\left(D_{2}^{r} c\right)(a, \Phi ; \delta \Phi)+R_{-N}(a, \Phi, \delta \Phi)$,

where

$$
\begin{gathered}
\left(D_{2}^{r} c\right)(a, \Phi ; \delta \Phi)=\sum_{|\alpha|=r} \frac{r !}{\alpha !}\left(\left(\partial^{\alpha} a\right) \circ \Phi\right) \cdot \delta \Phi^{\alpha} \\
R_{-N}(a, \Phi, \delta \Phi)=\sum_{|\alpha|=N+1} \frac{N+1}{\alpha !}(\delta \Phi)^{\alpha} \int_{0}^{1}(1-\theta)^{N}\left(\partial^{\alpha} a\right)(\Phi+\theta \cdot \delta \Phi) d \theta
\end{gathered}
$$

Then, the following sublemmas complete the smoothness of $c$.

SUBLEMMA 7.2. Under the above notations,

$$
\left(D_{2}^{r} c\right): \Sigma_{\mathbf{K}}^{-m} \times U_{\mathrm{id}} \times \Sigma_{\mathbf{R}}^{1} \cdots \times \Sigma_{\mathbf{R}}^{1} \rightarrow \Sigma_{\mathbf{K}}^{m}
$$

is continuous.

ProOF. Decompose $a \in \Sigma_{\mathbf{K}}^{-m}$ as 


$$
\begin{aligned}
& a=a_{-m}+a_{-m-1}+\cdots+a_{-L}, \\
& a_{-l} \in \kappa H_{\mathbf{K}}^{-l}\left(\mathbf{R}^{2 n}\right) \quad(m \leq l \leq L-1), \\
& a_{-L}=a-\sum_{l=m}^{L-1} a_{-m} \in \mathfrak{B}_{\mathbf{K}}^{-L}\left(\mathbf{R}^{2 n}\right) \quad(\text { cf. 3.3, 3.2). }
\end{aligned}
$$

For any $\alpha=\left(\alpha_{1}, \cdots, \alpha_{2 n}\right)$, and $-l(>-L)$, we have

$$
H_{\mathbf{K}}^{-1}\left(\mathbf{R}^{2 n}\right) \text {-term of }\left(D_{2}^{r} c\right)(a, \Phi ; \delta \Phi)=\left\{\sum^{\prime}\left(\delta \Phi_{i}\right)^{\alpha} \frac{1}{r !}\left(\Phi_{j}\right)^{\gamma}\left(\partial^{\alpha+\gamma} a_{k}\right) \circ \Phi_{1}\right\}(\omega) \rho^{-l} \text {, }
$$

where

$$
\begin{array}{cc}
\left(\delta \Phi_{i}\right)^{\alpha}=\left(\delta \Phi_{i_{1}}^{(1)}\right)^{\alpha_{1}} \cdots\left(\delta \Phi_{i_{2 n}}^{(2 n)}\right)^{\alpha_{2 n}} & \left(i_{l} \leq 1\right), \\
\left(\Phi_{j}\right)^{\gamma}=\left(\Phi_{j_{1}}^{(1)}\right)^{\gamma_{1}} \cdots\left(\Phi_{j_{2 n}}^{(2 n)}\right)^{\gamma_{2 n}} & \left(j_{l} \leq 0\right),
\end{array}
$$

and summation $\Sigma^{\prime}$ is taken for all multi-indices $(\alpha, \gamma, \boldsymbol{i}, \boldsymbol{j})$ satisfying

$$
-l=-|\gamma|-|\alpha|-k+\sum_{p=1}^{2 n} \gamma_{p} \cdot j_{p}+\sum_{p=1}^{2 n} \alpha_{p} \cdot i_{p}, \quad|\gamma| \leq L-1
$$

Since all terms of (7.8) are written by using higher derivatives of $a, \Phi, \delta \Phi$ and $a$ is smooth, we easily see the smoothness of (7.8).

On the other hand,

$$
\begin{aligned}
& \mathfrak{B}_{\mathbf{K}}^{-L}\left(\mathbf{R}^{2 n}\right) \text {-term of }\left(D_{2}^{r} c\right)(a, \Phi ; \delta \Phi) \\
& =(1-\kappa) \sum^{\prime}\left(\delta \Phi_{i}\right)^{\alpha} \frac{1}{r !}\left(\Phi_{j}\right)^{\gamma}\left(\partial^{\alpha+\gamma} a_{k}\right) \circ \Phi_{1} \\
& \quad+\sum_{|\gamma| \leq L-1} \sum^{\prime \prime}\left(\delta \Phi_{i}\right)^{\alpha} \frac{1}{r !}\left(\Phi_{j}\right)^{\gamma}\left(\partial^{\alpha+\gamma} a_{k}\right) \circ \Phi_{1} \\
& \quad+\sum_{|\gamma|=L} \sum^{\prime \prime} \frac{L}{\gamma !}\left(\Phi_{(0,-L)}\right)^{\gamma} \int_{0}^{1}(1-\tau)^{L-1}\left(\partial^{\alpha+\gamma} a_{k}\right) \circ\left(\Phi_{1}+\tau \Phi_{(0,-L)}\right) d \tau,
\end{aligned}
$$

where the summation $\sum^{\prime \prime}$ is taken over the multi-indices $(\alpha, \gamma, \boldsymbol{i}, \boldsymbol{j})$ satisfying

$$
-L=-|\gamma|-|\alpha|-k+\sum_{p=1}^{2 n} \gamma_{p} \cdot j_{p}+\sum_{p=1}^{2 n} \alpha_{p} \cdot i_{p} .
$$

We see that 


$$
\begin{aligned}
& \left(1+\rho^{2}\right)^{L / 2} \mid \partial^{\beta}(\text { r.h.s. of }(7.11)) \mid \\
& \leq C\left\{\sum^{\prime} \frac{1}{\gamma !}\left\|\delta \Phi_{i}\right\|_{i,|\beta|-|i|+1}^{\alpha}\left\|\Phi_{j}\right\|_{j,|\beta|-|j|+1}^{\gamma}\right. \\
& \times \llbracket\left(\partial^{\alpha+\gamma} a_{k}\right) \circ \Phi_{1} \prod_{k-|\alpha|-|\gamma|,|\beta|-k+|\alpha|+|\gamma|+1} \\
& +\sum_{|\gamma|<L} \sum^{\prime \prime} \frac{1}{\gamma !} \llbracket \delta \Phi_{i}\left\|_{i,|\beta|-|i|+1}^{\alpha}\right\| \Phi_{j} \|_{j,|\beta|-|j|+1}^{\gamma} \\
& \times \llbracket\left(\partial^{\alpha+\gamma} a_{k}\right) \circ \Phi_{1} \rrbracket_{k-|\alpha|-|\gamma|,|\beta|-k+|\alpha|+|\gamma|+1} \\
& +\sum_{|\gamma|=L} \sum^{\prime \prime} \frac{L}{\gamma !}\left\|\delta \Phi_{i}\right\|_{i,|\beta|-|i|+1}^{\alpha}\left\|\Phi_{j}\right\|_{j,|\beta|-|j|+1}^{\gamma} \\
& \left.\times \int_{0}^{1}(1-\tau)^{L-1} \llbracket\left(\partial^{\alpha+\gamma} a_{k}\right) \circ\left(\Phi_{1}+\tau \Phi_{(0,-L)}\right) \prod_{k-|\alpha|-|\gamma|,|\beta|-k+|\alpha|+|\gamma|+1} d \tau\right\},
\end{aligned}
$$

where

$$
\left\|\delta \Phi_{i}\right\|_{i, l}^{\alpha}=\prod_{k=1}^{2 n} \llbracket \delta \Phi_{i_{k}}^{(k)} \prod_{i_{k}, l}^{\alpha_{k}}, \quad\left\|\Phi_{j}\right\|_{j, l}^{\nu}=\prod_{k=1}^{2 n} \| \Phi_{j_{k}}^{(k)} \prod_{j_{k}, l}^{\gamma_{k}}
$$

Since Jacobian matrices of $\Phi_{1}$ and $\Phi_{1}+\tau\left(\Phi_{0}+\cdots+\Phi_{-L}\right)$ are close to $E_{2 n}$ uniformly on $\mathbf{R}^{2 n}$, we have

$$
\begin{aligned}
& \|\left(\partial^{\gamma} \partial^{\alpha} a_{k}\right) \circ\left(\Phi_{1}\right) \rrbracket_{k-|\alpha|-|\gamma|,|\beta|-k+|\alpha|+|\gamma|+1} \leq M \llbracket a_{k} \rrbracket_{k,|\beta|-k+|\alpha|+|\gamma|+1}, \\
& \llbracket\left(\partial^{\gamma} \partial^{\alpha} a_{k}\right) \circ\left(\Phi_{1}+\tau \Phi_{(0,-L)}\right) \prod_{k-|\alpha|-|\gamma|,|\beta|-k+|\alpha|+|\gamma|+1} \leq M \llbracket a_{k} \rrbracket_{k,|\beta|-k+|\alpha|+|\gamma|+1} .
\end{aligned}
$$

Therefore (7.13) is bounded by

$$
\begin{aligned}
& C\left\{\sum^{\prime} \llbracket \delta \Phi_{i} \rrbracket_{i,|\beta|-|i|+1}^{\alpha} \rrbracket \Phi_{j} \rrbracket_{j,|\beta|-|j|+1}^{\gamma} \llbracket a_{k} \rrbracket_{k,|\beta|-k+|\alpha|+|\gamma|+1}\right. \\
& \left.\sum_{|\gamma| \leq L} \sum^{\prime \prime} \llbracket \delta \Phi_{i}\left\|_{i,|\beta|-|i|+1}^{\alpha}\right\| \Phi_{j} \|_{j,|\beta|-|j|+1}^{\nu} \llbracket a_{k} \prod_{k,|\beta|-k+|\alpha|+|\gamma|+1}\right\} .
\end{aligned}
$$

SUBLEMMA 7.3. With the same notations as above, the remainder term

$$
\Gamma_{-N}(t, a, \Phi, \delta \Phi)= \begin{cases}0, & (t=0) \\ \left(1 / t^{N}\right) R_{-N}(a, \Phi, \delta \Phi), & (t \neq 0)\end{cases}
$$

is continuous.

PROOF. Remark that $H_{\mathbf{K}}^{-1}$-term of the non-trivial term $(t \neq 0)$ of $(7.16)$ is given by 


$$
\begin{aligned}
& \left\{\sum_{|\alpha|=N+1} \sum^{\prime} \frac{(N+1) t^{N+1}}{\alpha ! t^{N}}\left(\delta \Phi_{i}\right)^{\alpha}\right. \\
& \left.\times \int_{0}^{1}(1-\theta)^{N} \frac{1}{\gamma !}\left(\Phi_{j}+\theta t \delta \Phi_{j}\right)^{\gamma}\left(\partial^{\gamma} \partial^{\alpha} a_{k}\right)\left(\Phi_{1}+\theta t \delta \Phi_{1}\right) d \theta\right\}(\omega) \rho^{-l},
\end{aligned}
$$

where

$$
\left(\Phi_{j}+\theta t \delta \Phi_{j}\right)^{\gamma}=\prod_{p=1}^{2 n}\left(\Phi_{j_{p}}^{p}+\theta t \delta \Phi_{j_{p}}^{p}\right)^{\gamma_{p}}
$$

and the summation $\sum^{\prime}$ runs through the multi-indices given by (7.10). Since all terms are written by using higher derivatives of $\Phi, \delta \Phi, a$, we easily see the smoothness of (7.17).

On the other hand, the $\mathfrak{B}_{\mathbf{K}}^{-L}$-terms of the non-trivial term $(t \neq 0)$ of $(7.16)$ is given by

$$
\begin{aligned}
& (1-\kappa) \sum_{|\alpha|=N+1} \sum^{\prime} \frac{(N+1) t^{N+1}}{\alpha ! t^{N}}\left(\delta \Phi_{i}\right)^{\alpha} \\
& \quad \times \int_{0}^{1}(1-\theta)^{N} \frac{1}{\gamma !}\left(\Phi_{j}+\theta t \delta \Phi_{j}\right)^{\gamma}\left(\partial^{\gamma} \partial^{\alpha} a_{k}\right)\left(\Phi_{1}+\theta t \delta \Phi_{1}\right) d \theta \\
& +\sum_{|\alpha|=N+1}\left\{\sum_{|\gamma|<L} \sum^{\prime \prime} \frac{(N+1) t^{N+1}}{\alpha ! t^{N}}\left(\delta \Phi_{i}\right)^{\alpha} \int_{0}^{1}(1-\theta)^{N} \frac{1}{\gamma !}\left(\Phi_{j}+\theta t \delta \Phi_{j}\right)^{\gamma}\right. \\
& \quad \times\left(\partial^{\gamma} \partial^{\alpha} a_{k}\right)\left(\Phi_{1}+\theta t \delta \Phi_{1}\right) d \theta \\
& +\sum_{|\gamma|=L} \sum^{\prime \prime} \frac{(N+1) t^{N+1}}{\alpha ! t^{N}}\left(\delta \Phi_{i}\right)^{\alpha} \int_{0}^{1}(1-\theta)^{N} \frac{L}{\gamma !}\left(\Phi_{j}+\theta t \delta \Phi_{j}\right)^{\gamma} \\
& \left.\quad \times\left(\int_{0}^{1}\left((1-\tau)^{L-1} \partial^{\gamma+\alpha} a \circ\left(\Phi^{(1)}+\theta t \delta \Phi^{(1)}\right)+\tau\left\{\Phi_{(0,-L)}+\theta t \delta \Phi_{(0,-L)}\right\}\right) d \tau\right) d \theta\right\},
\end{aligned}
$$

where the summation $\sum^{\prime \prime}$ runs through the set of multi-indices given in (7.12). Jacobian matrices of

$$
\Phi_{1}+\theta t \delta \Phi_{1}, \quad\left(\Phi^{(1)}+\theta t \delta \Phi^{(1)}\right)+\tau\left\{\Phi_{(0,-L)}+\theta t \delta \Phi_{(0,-L)}\right\}
$$

are close to $E_{2 n}$ uniformly $\mathbf{R}^{2 n}$. By a similar argument for $D_{2} c$, we have

$$
\begin{aligned}
& \left(1+\rho^{2}\right)^{L / 2} \mid \partial^{\beta} \text { 1.h.s. of (7.18)| } \\
& \leq M t\left\{\sum^{\prime} \llbracket \delta \Phi_{i} \prod_{i,|\beta|-|i|+1}^{\alpha} \llbracket \Phi_{j} \rrbracket_{j,|\beta|-|j|+1}^{\gamma} \rrbracket a_{k} \rrbracket_{k,|\beta|-k+|\alpha|+|\gamma|+1}\right. \\
& \left.\quad+\sum_{|\nu| \leq L} \sum^{\prime \prime} \rrbracket \delta \Phi_{i} \prod_{i,|\beta|-|i|+1}^{\alpha} \llbracket \Phi_{j} \llbracket \gamma_{j,|\beta|-|j|+1} \llbracket a_{k} \rrbracket_{k,|\beta|-k+|\alpha|+|\gamma|+1}\right\} .
\end{aligned}
$$

Thus, we see $c$ is a smooth mapping. 
Next, we show the smoothness of the inverse.

LEMMA 7.4. Under the same notations and assumptions of Lemma 7.1, the mapping $U_{\text {id }} \ni \Phi \mapsto \Phi^{-1} \in \Sigma_{\mathbf{R}}^{1}\left(\mathbf{R}^{2 n}\right)$ is smooth.

PROOF. To see that $U_{\text {id }} \ni \Phi \mapsto \Phi^{-1} \in \Sigma_{\mathrm{R}}^{1}$ is a continuous mapping, it is sufficient to show the following estimate: For any $k$,

$$
\begin{aligned}
& \| \sum_{|\alpha|=1} \frac{1}{\alpha !}\left[-\delta \Phi(\Phi+\delta \Phi)^{-1}(z)\right]^{\alpha} \int_{0}^{1} \partial^{\alpha} \Phi^{(p)}\left(z-\theta \delta \Phi(\Phi+\delta \Phi)^{-1}(z)\right) d \theta \rrbracket_{-M, k} \\
& \leq C_{k, \Phi}\left\{\llbracket \delta \Phi^{(1)} \rrbracket_{-M, \delta_{k}}+\cdots+\llbracket \delta \Phi^{(2 n)} \rrbracket_{-M, \delta_{k}}\right\},
\end{aligned}
$$

where $\delta_{k}$ is a positive constant depending on $k, C_{k, \Phi}$ depends on $k, \Phi$ but it is independent of $\delta \Phi \in U$. By the formula of higher order derivatives of composition of functions, it is sufficient to show that if $\delta \Phi$ is sufficiently small, then

$$
\llbracket(\Phi+\delta \Phi)^{-1(p)} \rrbracket_{-M, k} \leq C .
$$

Here, we need the following fact:

Let $U, V$ be open in $\mathbf{R}^{n}$. If $\Psi: U \ni w \mapsto z \in V$ is a diffeomorphism, then $\partial_{z}^{\alpha}\left(\Psi^{-1}\right)$ have the following form:

$$
\sum_{\left|\delta_{1}\right|+\cdots+\left|\delta_{l}\right|=l+|\alpha|-1} C_{\delta_{1}, \cdots, \delta_{l}, i_{1}, \cdots, i_{l}} \frac{\left(\partial_{w}^{\delta_{1}} \Psi^{\left(i_{1}\right)} \cdots \partial_{w}^{\delta_{l}} \Psi^{\left(i_{l}\right)}\right) \circ \Psi^{-1}(z)}{\left[\operatorname{det}(\partial \Psi / \partial w) \circ \Psi^{-1}(z)\right]^{p_{\delta_{1}}, \ldots, \delta_{l}, i_{1}, \ldots, i_{l}}} .
$$

In (7.23), $l-\left|\delta_{1}\right|-\left|\delta_{2}\right|-\cdots-\left|\delta_{l}\right|=1-|\alpha|$ holds. There exists positive number $\varepsilon$ $(>0)$ such that if $\delta \Phi$ is sufficiently close to 0 then the following estimate holds:

$$
1-\varepsilon \leq|\operatorname{det} D(\Phi+\delta \Phi)| \leq 1+\varepsilon .
$$

Therefore, we obtain that

$$
\llbracket(\Phi+\delta \Phi)^{-1} \rrbracket_{-M, k} \leq C \quad(k \geq M+1) .
$$

Using the continuity of the mapping, we next show that the mapping $\Phi \mapsto \Phi^{-1}$ is $C^{1}$. Put $c(\Psi, \Phi)=\Psi \circ \Phi, i(\Psi)=\Psi^{-1}$. Then we have

$$
\begin{aligned}
& {\left[c(i(\Phi+\delta \Phi), \Phi)-c(i(\Phi), \Phi)-\left(-D_{2} c(i(\Phi), \Phi)\right)(\delta \Phi)\right] \circ \Phi^{-1}} \\
& =\left[\left(-D_{2} c(i(\Phi+\delta \Phi), \Phi)\right)(\delta \Phi)+D_{2} c(i(\Phi), \Phi)(\delta \Phi)\right. \\
& \left.\quad-\int_{0}^{1}(1-\theta)\left(D_{2}^{2} c(i(\Phi+\delta \Phi), \Phi+\theta \delta \Phi)\right)(\delta \Phi, \delta \Phi) d \theta\right] \circ \Phi^{-1} .
\end{aligned}
$$

As $c$ is $C^{\infty}$, we see that $i$ is $C^{1}$ and

$$
\left.(D i)(\Phi, \delta \Phi)=\left[-D_{2} c(i(\Phi), \Phi)(\delta \Phi)\right] \circ \Phi^{-1}=c\left(-D_{2} c(i(\Phi), \Phi)\right)(\delta \Phi), i(\Phi)\right) .
$$

Successively, we see that $i$ is $C^{r}$ for any $r$, i.e. $i$ is $C^{\infty}$. 
Using the above lemmas, we see also the following lemma.

LEMMA 7.5. The correspondence $S=S_{2}+S_{1} \mapsto \hat{S}=\hat{S}_{2}+\hat{S}_{1}$ given in (5.35) is a smooth mapping of $U_{x \cdot \xi}$ into $H_{\mathbf{R}}^{2} \oplus H_{\mathbf{R}}^{1}$.

Proof. The above lemma asserts that the second mapping of the following diagram is a smooth mapping:

$$
S_{2}+S_{1} \mapsto \chi_{s_{2}+s_{1}} \mapsto \chi \chi_{S_{2}+s_{1}}^{-1} \mapsto \sigma_{\chi_{s_{2}+s_{1}}^{-1}} \mapsto \hat{S}_{2}+\hat{S}_{1}
$$

Since we use only Lemma 5.2 at the third mapping, it is obvious that the third mapping is smooth.

As we have seen in $\S 5, \S 6$, multiplication on $G F^{0} / G \Sigma^{0}$ is given as follows:

$$
\begin{aligned}
& \left(F\left(a, S_{1}, \tilde{\varphi}_{S_{2}}\right) \circ G \Sigma^{0}\right) \circ\left(F\left(b, T_{1}, \tilde{\varphi}_{T_{2}}\right) \circ G \Sigma^{0}\right) \\
& \quad=F\left(c,\left\{\left(S_{2}+S_{1}\right) \odot\left(T_{2}+T_{1}\right)\right\}_{1}, \tilde{\varphi}_{\left\{\left(s_{2}+S_{1}\right) \odot\left(T_{2}+T_{1}\right)\right\}_{2}}\right) \circ G \Sigma^{0} \\
& \quad=F\left(c,\left\{\left(S_{2}+S_{1}\right) \odot\left(T_{2}+T_{1}\right)\right\}_{1}, \tilde{\varphi}_{S_{2} \odot T_{2}}\right) \circ G \Sigma^{0} .
\end{aligned}
$$

As for $\left\{\left(S_{2}+S_{1}\right) \odot\left(T_{2}+T_{1}\right)\right\}_{2}$ and $\left\{\left(S_{2}+S_{1}\right) \odot\left(T_{2}+T_{1}\right)\right\}_{1}$, see (5.33) in the proof of Lemma 5.3. Especially, we see

$$
\left\{S_{2} \odot T_{2}\right\}_{2}=\left\{\left(S_{2}+S_{1}\right) \odot\left(T_{2}+T_{1}\right)\right\}_{2} .
$$

To show the smoothness of the multiplication of generating functions, we have to show the following lemma by using Lemmas 7.1, 5.5 and 7.4

LEMMA 7.6. Under the same notations and assumptions as in Lemma 7.1, $F$ defined in (5.31) and the following mapping are smooth:

$$
U_{x \cdot \xi} \times U_{x \cdot \xi} \ni(S, T) \mapsto S \odot T \in \Sigma_{\mathbf{R}}^{2},
$$

where $U_{x \cdot \xi}$ is a certain neighborhood of $x \cdot \xi$ in $H_{\mathbf{R}}^{2} \oplus H_{\mathbf{R}}^{1}$.

Proof. First of all, we shall show the smoothness of $F$ of (5.31). Since $\hat{\Phi}$ in (5.17) depends on $S, T \in U_{x \cdot \xi}$, we denote this by $\Phi_{(S, T)}$ also. If we put

$$
\delta \Phi_{(S, T)}=\Phi_{(S+\delta S, T+\delta T)}-\Phi_{(S, T)}=\left(\begin{array}{c}
0 \\
\nabla_{\bar{x}} \delta T \\
\nabla_{\bar{\xi}} \delta S \\
0
\end{array}\right),
$$

then we obtain

$$
\left(\Phi_{(S, T)}+\delta \Phi_{(S, T)}\right)^{-1}\left(\begin{array}{c}
\bar{x} \\
0 \\
0 \\
\xi
\end{array}\right)=\Phi_{(S, T)}^{-1}\left(\begin{array}{c}
\bar{x} \\
0 \\
0 \\
\xi
\end{array}\right)
$$




$$
-D_{(\bar{x}, \bar{\eta}, \bar{y}, \xi)} \Phi_{(S, T)}^{-1} \cdot\left(\delta \Phi_{(S, T)} \circ \Phi_{(S, T)}^{-1}\right)\left(\begin{array}{c}
\bar{x} \\
0 \\
0 \\
\xi
\end{array}\right)+R_{2}\left(\delta \Phi_{(S, T)}, \delta \Phi_{(S, T)}\right)\left(\begin{array}{c}
\bar{x} \\
0 \\
0 \\
\xi
\end{array}\right) .
$$

By a similar argument as in (7.25) of proof of Lemma 7.4, we see $\Phi_{(S, T)}$ is $C^{\mathbf{1}}$. Recall that

$$
\begin{aligned}
\delta \Phi_{(S, T)}\left(\begin{array}{c}
\bar{x} \\
\bar{\xi} \\
\bar{x} \\
\xi
\end{array}\right)=\left(\begin{array}{c}
0 \\
\nabla_{\bar{x}}(\delta T(\overline{\bar{x}}, \xi)) \\
\nabla_{\bar{\xi}}(\delta S(\bar{x}, \bar{\xi})) \\
0
\end{array}\right), \\
D_{(\bar{x}, \bar{\xi}, \bar{x}, \xi)} \Phi_{(S, T)}=\left(\begin{array}{cccc}
E_{n} & 0 & 0 & 0 \\
0 & -E_{n} & \partial_{\bar{x}} \partial_{\bar{x}} T & \partial_{\bar{x}} \partial_{\xi} T \\
\partial_{\bar{x}} \partial_{\xi} S & \partial_{\xi} \partial_{\xi} S & -E_{n} & 0 \\
0 & 0 & 0 & E_{n}
\end{array}\right) .
\end{aligned}
$$

By $D\left(\Phi^{-1}\right)=(D \Phi)^{-1} \circ \Phi^{-1}$, we have

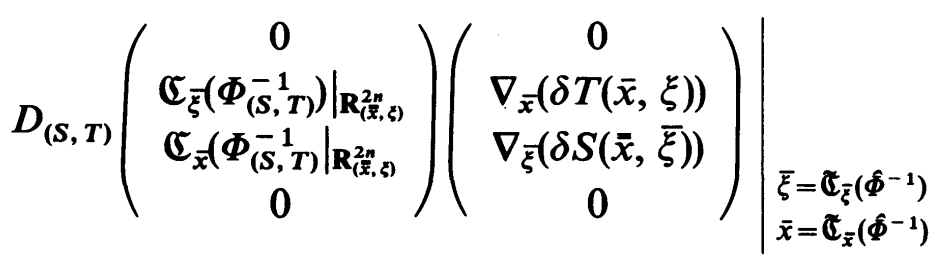

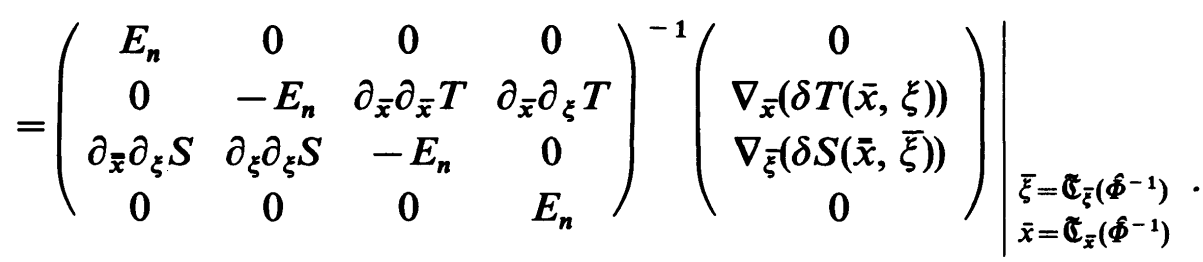

By Proposition 2.1, Lemmas 7.1 and 7.4, the right hand side of (7.32) is $C^{1}$, and hence so is the left hand side of (7.32). Thus we see $(S, T) \mapsto F(S, T)$ given by

$$
F(S, T)(\bar{x}, \xi)=\left(\widetilde{\mathbb{E}}_{\bar{\xi}}\left(\hat{\Phi}^{-1}\right), \widetilde{\mathbb{C}}_{\bar{x}}\left(\hat{\Phi}^{-1}\right)\right)
$$

is $C^{2}$ with respect to $(S, T)$. By induction, we see the mapping $F$ is smooth.

Similarly we can see that the following mappings

$$
\begin{gathered}
G:(S, T) \mapsto G(S, T) \in \Sigma_{\mathrm{R}}^{1}, \\
H:(S, T) \mapsto H(S, T) \in \Sigma_{\mathrm{R}}^{1}
\end{gathered}
$$

defined by 


$$
\begin{gathered}
(G(S, T))(\bar{x}, \xi)=\left(\bar{x}, \widetilde{\mathbb{C}}_{\bar{\xi}}\left(\hat{\Phi}^{-1}\right)\right), \\
(H(S, T))(\bar{x}, \xi)=\left(\widetilde{\mathbb{C}}_{\bar{x}}\left(\hat{\Phi}^{-1}\right), \xi\right)
\end{gathered}
$$

are smooth. Note that

$$
S \odot T=S \circ G(S, T)-\left\langle\widetilde{\mathfrak{C}}_{\bar{\xi}}\left(\hat{\Phi}^{-1}\right) \mid \widetilde{\mathfrak{C}}_{\bar{\xi}}\left(\hat{\Phi}^{-1}\right)\right\rangle+T \circ H(S, T) .
$$

Applying Lemma 7.1 to $S, T \in \Sigma_{\mathbf{R}}^{2}$ and the mapping stated above, we see that the mapping (7.6) is smooth.

(i) of Proposition B has already seen in Lemma 6.3.

Proof of Proposition B (ii), (iii). As we have seen in $\S 6$, we obtain that the symbol of composition (6.15) of two Fourier integral operators is given by

$$
\begin{aligned}
& c(\overline{\bar{x}}, \xi)=c\left(a, S_{1}, \tilde{\varphi}_{S_{2}}, b, T_{1}, \tilde{\varphi}_{T_{2}}\right)(\overline{\bar{x}}, \xi)
\end{aligned}
$$

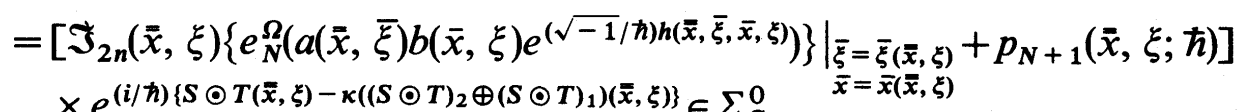

where $\Omega$ is given by (6.9). If we set

$$
e_{(N+1)}(\theta)=e^{\sqrt{-1} \theta}-e_{N}^{\sqrt{-1} \theta}
$$

then $p_{N+1}$ is written as follows (cf. $\left.[\mathrm{AF}]\right)$ :

$$
\begin{aligned}
p_{N+1}(\bar{x}, \xi) & =\hbar^{2 / 3} \iiint \iint_{\mathbf{R}^{2 n}} a\left(\bar{x}, \bar{\xi}(\bar{x}, \xi)+\hbar^{1 / 3} \bar{\eta}\right) b\left(\bar{\xi}(\bar{x}, \xi)+\hbar^{1 / 3} \bar{y}, \xi\right)(1-\kappa)(\bar{\eta}, \bar{y}) \\
& \left.\times e^{-\sqrt{-1}(\bar{\eta}, \bar{y}) \cdot(\bar{\sigma}, \bar{w})} e_{(N+1)}\left(-\frac{1}{2} \hbar^{1 / 3}\left\langle H^{-1}(\bar{x}, \xi)(\bar{\eta}, \bar{y}),(\bar{\eta}, \bar{y})\right\rangle\right) \bar{d} \bar{\eta} \bar{d} \bar{y}\right\} \bar{\sigma} \bar{d} \bar{d} \bar{w} \\
+ & \hbar^{2 / 3}\left\{\iint_{\mathbf{R}^{2 n}} a\left(\bar{x}, \bar{\xi}(\bar{x}, \xi)+\hbar^{1 / 3} \bar{\eta}\right) \cdot b\left(\bar{\xi}(\bar{x}, \xi)+\hbar^{1 / 3} \bar{y}, \xi\right) \kappa(\bar{\eta}, \bar{y})\right. \\
& \left.\times e^{(\sqrt{-1} / \hbar)\left\{S\left(\bar{x}, \bar{\xi}(\bar{x}, \xi)+\hbar^{1 / 3} \bar{\eta}\right)-\left(\bar{\xi}(\bar{x}, \xi)+\hbar^{1 / 3} \bar{\eta}\right) \cdot\left(\bar{x}(\bar{x}, \xi)+\hbar^{1 / 3} \bar{y}+T\left(\bar{x}(\bar{x}, \xi)+\hbar^{1 / 3} \bar{y}, \xi\right)\right\}\right.} d \bar{\eta} \bar{y} \bar{y}\right\} .
\end{aligned}
$$

The generating function $(S \odot T)_{2} \oplus(S \odot T)_{1}$ is an element of $H_{\mathbf{R}}^{2} \oplus H_{\mathbf{R}}^{1}$, and

$$
F\left(a, S_{1}, \tilde{\varphi}_{S_{2}}\right) \circ F\left(b, T_{1}, \hat{\varphi}_{T_{2}}\right)=F\left(c(\bar{x}, \xi),(S \odot T)_{1}, \tilde{\varphi}_{(S \odot T)_{2}}\right) .
$$

As for the phase part $(S \odot T)_{1}, \tilde{\varphi}_{(S \odot T)_{2}}$, the rule of composition is the same as in the group $G F^{0} / G \Sigma^{0}$. The smoothness of the phase part has been proved in Lemma 7.6.

As for symbol part $c$, we shall split $H_{\mathbf{C}}^{i}$-component and $\mathfrak{B}_{\mathbf{C}}^{-\boldsymbol{L}}$-component. By applying Lemmas 7.1, 7.4 and 7.6, we can prove the smoothness of $H_{\mathbf{C}}^{i}$-component. On the other hand, by using the Taylor's theorem and Lemmas 7.1, 7.4 and 7.6 to $p_{N+1}$ (cf. (7.39)), we see the smoothness of $\mathfrak{B}_{\mathbf{C}}^{-L}$-component. Therefore, we see smoothness of composition (ii). 
By using (ii) and Lemma 6.5, we see the smoothness of inverse (iii).

\section{References}

[AH] S. Albeverio and R. J. HøEGH-KROHN, Mathematical Theory of Feynman Path Integral, Lecture Notes in Math. 523 (1976), Springer.

[AF] K. Asada and D. Fujiwara, On some oscillatory integral transformations in $L^{2}\left(\mathbf{R}^{n}\right)$, Japan. J. Math. 4 (1978), 299-361.

[ASR1] M. AdAms, T. Ratiu and R. Schmid, A Lie group structure for pseudodifferential operators, Math. Ann. 273 (1986), 529-551.

[ASR2] M. AdAmS, T. RATIU and R. Schmid, A Lie group structure for Fourier integral operators, Math. Ann. 276 (1986), 19-41.

[FH] R. P. Feynman and A. R. HibBs, Quantum Mechanics and Path Integrals, Mcgraw-Hill (1965).

[F1] D. Fujiwara, Asymptotic Methods in Linear Partial Differential Equations, Iwanami (1977) (in Japanese).

[F2] D. Fujiwara, A construction of the fundamental solution for the Schrödinger equation, J. Analyse Math. 35 (1979), 41-96.

[F3] D. FujIWARA, Remarks on convergence of some Feynman path integrals, Duke Math. J. 47 (1980), 559-600.

[I1] K. Ito, Wiener integral and Feynman integral, Proc. of the Fourth Berkeley Sympos. on Math. Stat. and Probab. vol. 2, Univ. California Press (1961), 227-238.

[12] K. Iто, Generalized uniform complex measure in Hilbert space and its application to the Feynman path integrals, Proc. of the Fifth Berkeley Sympos. on Math. Stat. and Probab. vol. 2, part 1, Univ. California Press (1967), 145-161.

[IM] A. INOUE and Y. MAEDA, On integral transformations associated with a certain Lagrangian - as a prototype of quantization, J. Math. Soc. Japan 37 (1985), 219-244.

[Ki] H. KiTADA, On a construction of the fundamental solution for Schrödinger equations, J. Fac. Sci. Univ. Tokyo Sect. IA Math. 27 (1980), 193-226.

[KK] H. Kitada and H. KumanoGo, A family of Fourier integral operators and the fundamental solution for a Schrödinger equation, Osaka J. Math. 18 (1981), 291-360.

[Ku] H. Kumanogo, Pseudo Differential Operators, MIT Press (1982).

[L] J. A. LeSLIE, Some Frobenius theorems in global analysis, J. Differential Geometry 2 (1968), 279-297.

[N1] E. Nelson, Topics in Dynamics I: Flows, Princeton Univ. Press (1969).

[N2] E. Nelson, Feynman integrals and Schrödinger equation, J. Math. Phys. 5 (1964), 332-343.

[Om] H. OMORI, Theory of Infinite Dimensional Lie Groups, Kinokuniya (1978) (in Japanese).

[OMY] H. OMORI, Y. MAedA and A. YoshiokA, On regular Fréchet-Lie groups, I, II, Tokyo J. Math. 3 (1980), 353-390; 4 (1981), 231-253.

[OMYK] H. OMORI, Y. MAeda, A. YoshioKa and O. Kobayashi, On regular Fréchet-Lie groups III, IV, V, VI, VII, VIII, Tokyo J. Math. 4 (1981), 255-277; 5 (1982), 365-398; 6 (1983), 39-64; 6 (1983), 217-246; 7 (1984), 315-336; 8 (1985), 1-47.

[S] J. T. Schwartz, Non-Linear Functional Analysis, Gordon and Breach (1969).

Present Address:

DePartment of Mathematics, Science University of TOKyo, Noda, Chiba, 278 Japan. 